\title{
A HARPS RV search for planets around young nearby stars ${ }^{\star}$
}

\author{
A. Grandjean ${ }^{1}$, A.-M. Lagrange ${ }^{1}$, M. Keppler ${ }^{2}$, N. Meunier ${ }^{1}$, L. Mignon ${ }^{1}$, S. Borgniet $^{3}$, G. Chauvin ${ }^{1}$, S. Desidera ${ }^{4}$, \\ F. Galland ${ }^{1}$, S. Messina ${ }^{5}$, M. Sterzik ${ }^{6}$, B. Pantoja ${ }^{7}$, L. Rodet ${ }^{1}$, and N. Zicher ${ }^{8}$
}

${ }^{1}$ Univ. Grenoble Alpes, CNRS, IPAG, 38000 Grenoble, France
e-mail: antoine.grandj ean1@univ-grenoble-alpes.fr
2 Max Planck Institute for Astronomy, Königstuhl 17, 69117 Heidelberg, Germany
${ }^{3}$ CNRS Lesia (UMR8109) - Observatoire de Paris, Paris, France
${ }^{4}$ INAF - Osservatorio Astronomico di Padova, Vicolo dell'Osservatorio 5, Padova 35122, Italy
${ }^{5}$ INAF - Osservatorio Astrofisico di Catania, via Santa Sofia, 78 Catania, Italy
${ }^{6}$ European Southern Observatory (ESO), Alonso de Córdova 3107, Vitacura, Casilla 19001, Santiago, Chile
7 Departamento de Astronomía, Universidad de Chile, Camino al Observatorio, Cerro Calán, Santiago, Chile
${ }^{8}$ Oxford Astrophysics, Department of Physics, Denys Wilkinson Building, UK

Received 6 June 2019 / Accepted 14 October 2019

\begin{abstract}
Context. Young nearby stars are good candidates in the search for planets with both radial velocity (RV) and direct imaging techniques. This, in turn, allows for the computation of the giant planet occurrence rates at all separations. The RV search around young stars is a challenge as they are generally faster rotators than older stars of similar spectral types and they exhibit signatures of magnetic activity (spots) or pulsation in their RV time series. Specific analyses are necessary to characterize, and possibly correct for, this activity.

Aims. Our aim is to search for planets around young nearby stars and to estimate the giant planet (GP) occurrence rates for periods up to 1000 days.

Methods. We used the HARPS spectrograph on the $3.6 \mathrm{~m}$ telescope at La Silla Observatory to observe $89 A-M$ young $(<600 \mathrm{Myr})$ stars. We used our SAFIR (Spectroscopic data via Analysis of the Fourier Interspectrum Radial velocities) software to compute the RV and other spectroscopic observables. Then, we computed the companion occurrence rates on this sample.

Results. We confirm the binary nature of HD 177171, HD 181321 and HD 186704 . We report the detection of a close low mass stellar companion for HIP 36985. No planetary companion was detected. We obtain upper limits on the GP ( $\left.<13 M_{\mathrm{Jup}}\right)$ and BD $\left(\in[13 ; 80] M_{\mathrm{Jup}}\right)$ occurrence rates based on 83 young stars for periods less than 1000 days, which are set, $2_{-2}^{+3}$ and $1_{-1}^{+3} \%$.
\end{abstract}

Key words. techniques: radial velocities - stars: activity - binaries: spectroscopic - planetary systems - starspots stars: variables: general

\section{Introduction}

More than four thousand exoplanets have been confirmed and most of them have been found by transit or radial velocity (RV) techniques ${ }^{1}$. The latter, although very powerful, is limited by the parent star's activity (spots, plages, convection, and pulsations). Young stars are generally faster rotators than their older counterparts (Stauffer et al. 2016; Rebull et al. 2016; Gallet \& Bouvier 2015). They can also exhibit activity-induced RV jitter with amplitudes up to $1 \mathrm{~km} \mathrm{~s}^{-1}$ (Lagrange et al. 2013), larger than the planet's induced signal. False positive detections have been reported around young stars in the past (Huélamo et al. 2008; Figueira et al. 2010; Soto et al. 2015).

We have carried out an RV survey to search for planets around such young stars with the High Accuracy Radial velocity Planet Searcher (HARPS; Mayor et al. 2003) and SOPHIE (Bouchy \& Sophie Team 2006) spectrographs with the final aim of coupling RV data with direct imaging (DI) data, which will allow for the computation of detection limits, for each targets at

\footnotetext{
* A table of the radial velocities is only available at the CDS via anonymous ftp to cdsarc.u-strasbg. fr (130.79.128.5) or via http:// cdsarc.u-strasbg.fr/viz-bin/cat/J/A+A/633/A44

1 exoplanet.eu
}

all separations and then to compute occurrences rates for all separations. A feasibility study was carried out by Lagrange et al. (2013) on 26 stars of the survey with HARPS, demonstrating that we can probe for giant planets $\left(1-13 M_{\text {Jup }}\right.$, hereafter GP) with semi-major axis up to 2 au and couple the survey data with direct imaging data. The time baseline of our survey also permits a probe of the hot Jupiter (hereafter HJ) domain around young stars. Although GP formation models predict a formation at a few au (Pollack et al. 1996), migration through disc-planet interaction (Kley \& Nelson 2012) or gravitational interaction with a third body can allow the planet to finally orbit close to the star (Teyssandier et al. 2019). HJ are common among exoplanets orbiting older main sequence stars as they represent one detected planet out of five (Wright et al. 2012). While previous RV surveys on small sets of young stars showed no evidence for the presence of young HJ (Esposito et al. 2006; Paulson \& Yelda 2006), two $\mathrm{HJ}$ around young stars were recently discovered by Donati et al. (2016) and Yu et al. (2017). However, we still need to find out if this kind of object is common at young age or not and we need to compare the migration models with observations in order to constrain the migration timescales.

Here, we report on the results of our large HARPS survey. We describe our survey sample and observations in Sect. 2. In Sect. 3, we focus on GP, brown dwarf (BD), and stellar 


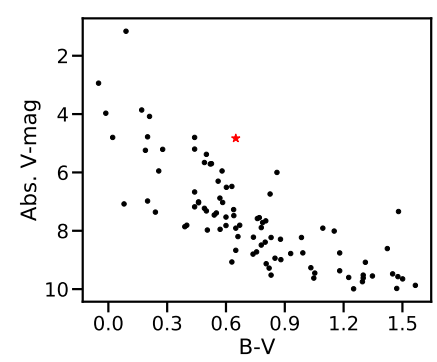

(a)

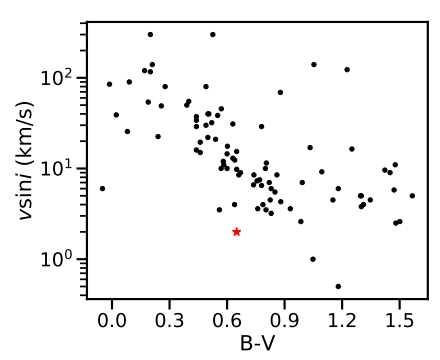

(b)

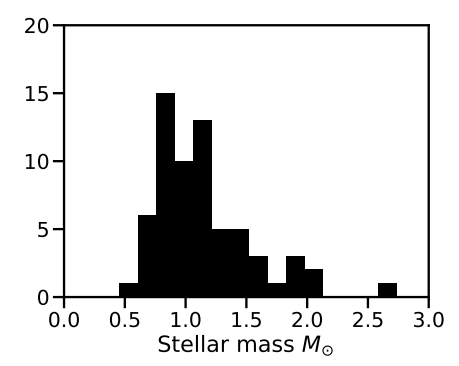

(c)

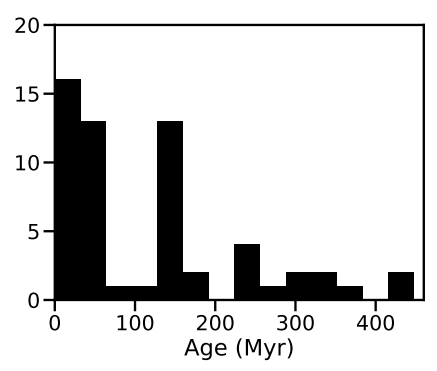

(d)

Fig. 1. Main physical properties of our sample. (a) Absolute $V$-magnitude vs. $B-V$. Each black dot corresponds to one target. The Sun is displayed (red star) for comparison. (b) $v \sin i$ vs $B-V$ distribution. (c) Mass histogram (in $M_{\odot}$ ). (d) Age histogram.

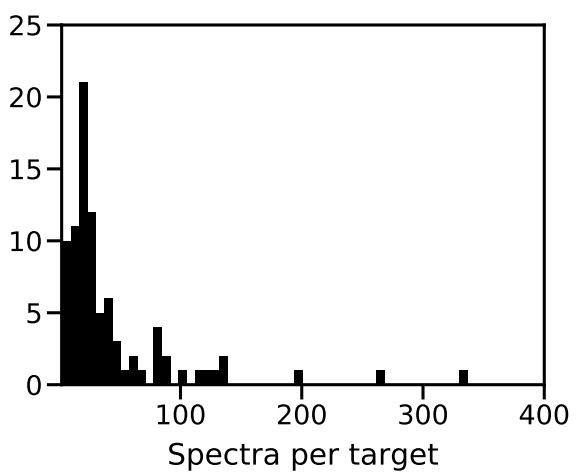

(a)

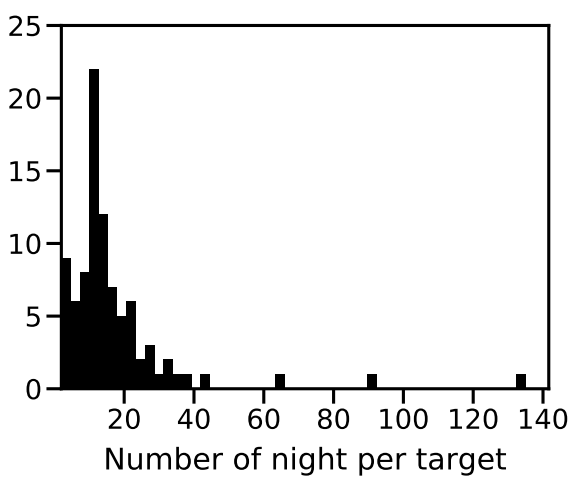

(b)

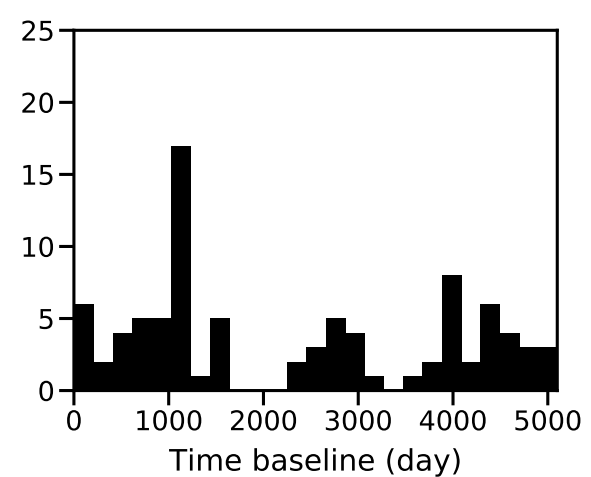

(c)

Fig. 2. Observation summary. (a) Histogram of the number of spectra per target, excluding HD 216956 (Fomalhaut, 834 spectra) and HD 039060 $(\beta$ Pic, 5108 spectra). (b) Histogram of the number of nights per target. (c) Histogram of the time baselines.

companions detections. In Sect. 4, we perform a statistical analysis of our sample and compute the close GP and BD occurrence rates around young stars. We conclude our findings in Sect. 5.

\section{Description of the survey}

\subsection{Sample}

We selected a sample of 89 stars, chosen to their brightness $(V<10)$, ages as fund in the literature $(<300 \mathrm{Myr}$ for most of them, see Table A.1), and distance $(<80 \mathrm{pc})$ determined from their HIPPARCOS parallaxes (van Leeuwen 2007). These criteria ensure we will get the best detection limits for both the HARPS RV and SPHERE DI surveys at, respectively, short (typically 2-5 au), and large (further than typically $5 \mathrm{au}$ ) separations. Indeed, observing bright stars allow us to obtain spectra with a better signal-to-noise ratio $(\mathrm{S} / \mathrm{N})$. Moreover, young planets are still warm from their formation and are then brighter, which lowers the contrast between them and their host stars, while short distances allow for better angular resolutions in direct imaging. Most of the targets are part of the SPHERE GTO The SpHere INfrared survey for Exoplanets (SHINE) survey sample (Chauvin et al. 2017a). Binary stars with an angular separation on the sky that is lower than 2 as were not selected to avoid contamination in the spectra from the companion

Their spectral types range from A0V to M5V (Fig. 1). Their projected rotational velocity $(v \sin i)$ range from 0.5 to $300 \mathrm{~km} \mathrm{~s}^{-1}$ with a median of $11 \mathrm{~km} \mathrm{~s}^{-1}$, their $V$-band magnitude are mainly between 4 and 10 with a median of 7.8. Their masses are between 0.6 and $2.74 M_{\odot}$ with median of $1.07 M_{\odot}$. Our sample includes 26 targets between $\mathrm{A} 0$ and $\mathrm{F} 5 \mathrm{~V}(B-V \in[-0.05: 0.52])$,
55 between $\mathrm{F} 6$ and $\mathrm{K} 5(B-V \in[-0.05: 0.52])$, and 8 between $\mathrm{K} 6$ and M5 $(B-V \geq 1.33)$. Noticeably, our sample includes stars with imaged planetary or substellar companions (among them $\beta$ Pic, AB Pic, HN Peg, GJ 504, HR 8799, HD 95086, HD 106906 or PZ Tel). We present the main characteristics of our star sample in Fig. 1 and Table A.1.

\subsection{Observations}

We observed our 89 targets mainly between 2013 and 2016. Some stars were part of previous surveys by Borgniet et al. (2017) and Lagrange et al. (2009a), which allows us to reach a time baseline up to $10 \mathrm{yr}$. Some stars had already been observed with HARPS before, some since the HARPS commissioning in 2003. Additional observations were also obtained in October 2017, December 2017, and March 2018.

We use the observing strategy described in Borgniet et al. (2017), which consist of recording two spectra per visit and to observe each target on several consecutive nights to have a good sampling of the short-term jitter. The median time baseline is 1639 days (mean time baseline of 2324 days), with a median number of spectra per target of 25 (52 on average) spaced on a median number of 12 nights (17 on average, Fig. 2). Details can be found in Table A.1.

\subsection{Observables}

From the HARPS spectra we derived the RV and whenever possible the cross-correlation function (CCF), bisector velocity span (hereafter BVS), and $\log R_{\mathrm{HK}}^{\prime}$ with our SAFIR software for Spectroscopic data via Analysis of the Fourier Interspectrum Radial 
velocities. It builds a reference spectrum from the median of all spectra available on a given star and computes the relative $\mathrm{RV}$ in the Fourier plane. The computed RV are then relative to the reference spectrum. The efficiency of this method was proved in the search of companions (Galland et al. 2005a,b). We mainly use the correlation between RV and BVS to determine the main source of RV variability: magnetic activity, pulsations or companions (Lagrange et al. 2009a; Borgniet et al. 2017). We excluded spectra with $\mathrm{S} / \mathrm{N}$ at $550 \mathrm{~nm}$ which was too high $(>380)$, to avoid saturation or too low $(<80)$, to avoid bad data, as well as spectra with an air mass that was too high ( $\sec z>3$ ), and spectra that were too different from the reference spectrum of the star $\left(\chi^{2}>10\right)$. For M-type stars, we used a lower limit of 40 in $\mathrm{S} / \mathrm{N}$ at $550 \mathrm{~nm}$ as it is a better compromise for these stars to provide enough spectra to perform our analysis without including bad spectra.

\section{Detected companions in the HARPS survey}

\subsection{Long period companions, RV long-term trends, and stellar binaries}

In this section we describe the stars for which we identified a GP companion with a period higher than 1000 days, a long-term trend RV signal or a binary signal. When possible, we characterize the companion using the yorbit software that fits RV with a sum of Keplerian or a sum of keplerians plus a drift (Ségransan et al. 2011).

\subsubsection{HD 39060}

$\beta$ Pic is an A6V pulsating star that hosts an imaged edge-on debris (Smith \& Terrile 1984) and gas disk (Hobbs et al. 1985), and an imaged GP at 9 au (Lagrange et al. 2009b, 2019a). This star presents also exocomets signatures in its spectra (LagrangeHenri et al. 1988; Beust et al. 1989; Kiefer et al. 2014). $\beta$ Pic was observed with HARPS since its commissioning in 2003, totalizing more than 6000 spectra with a mean S/N at $550 \mathrm{~nm}$ of 273. Until 2008, spectra were taken to study the Ca II absorption lines associated to the falling exocomets. Since 2008, we adopted a specific observation strategy, to properly sample the stellar pulsations and to correct the RV from them, (Lagrange et al. 2012, 2019b). It consisted in observing the star for continuous sequences of $1-2 \mathrm{~h}$. Longer sequences up to $6 \mathrm{~h}$ were obtained in 2017-2018. This allowed to detect a new GP companion within the pulsations signal of $\beta$ Pic. The discovery of this $10 M_{\text {Jup }}, 1200$ days period companion is detailed in Lagrange et al. (2019b).

\subsubsection{HD 106906}

HD 106906 is a F5V star in the Sco-Cen young association. Bailey et al. (2014) Imaged a giant planet companion at 7.1 as (650 au) in 2014. HARPS data from this survey together with the PIONER interferometer data allowed to detect a close low mass stellar companion to HD 106906 with a period of 10 days, (Lagrange et al. 2015). The presence of the binary could explain the wide orbit of HD $106906 \mathrm{~b}$ under some circumstances (Rodet et al. 2017).

\subsubsection{HD 131399}

HD 131399 is member of a complex hierarchical system. HD 131399A forms a binary with the tight binary HD 131399BC.
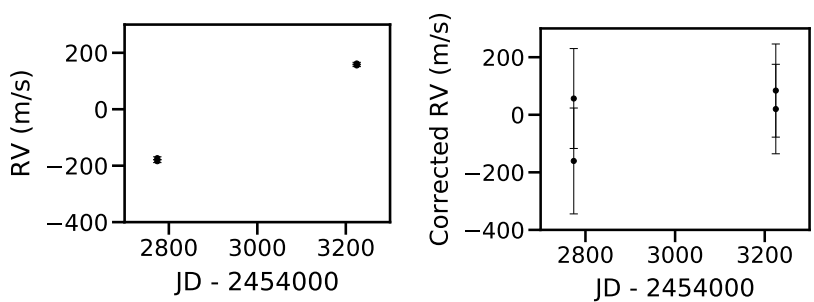

Fig. 3. HD $186704 \mathrm{RV}$ long term trend. Left: RV time variations, right: $\mathrm{RV}$ corrected from the RV-BVS correlation time variations.

A GP companion was discovered with SPHERE around HD 131399A by Wagner et al. (2016) but is now identified as a background star with similar proper motion (Nielsen et al. 2017). We detected in this survey the presence of a close stellar companion to HD 131399A, with a period of 10 days (0.1 au), and an $M \sin i$ of $450 M_{\text {Jup }}$ (Lagrange et al. 2017).

\subsubsection{HD 177171}

HD 177171 ( $\rho$ Tel) was reported as a spectroscopic binary by Nordström et al. (2004) and as an astrometric binary by Frankowski \& Jorissen (2007) from the HIPPARCOS data. This is confirmed by Lagrange et al. (2013) in the feasibility study of this survey. We measure an amplitude of at least $20 \mathrm{~km} \mathrm{~s}^{-1}$ in the RV. Our time sampling does not allow for the estimation the period of this stellar companion.

\subsubsection{HD 186704A}

HD 186704 is a known binary system with a companion at 10 as (Zuckerman et al. 2013). Nidever et al. (2002) report a trend in the RV of $88 \pm 8 \mathrm{~ms}^{-1} \mathrm{~d}^{-1}$ with a negative curvature based on 4 observations spaced on 70 days for HD 186704AB. Tremko et al. (2010) observe a change in the RV of $4200 \mathrm{~ms}^{-1}$ in 8682 days on HD 186704A. Finally, Tokovinin (2014) reporte HD 186704A as hosting a spectroscopic binary (SB) companion with a 3990 days period. We observe a trend of $340 \mathrm{~ms}^{-1}$ over a duration of 450 days in the RV of HD 186704A based on 4 spectra. This corresponds to a slope of $0.75 \mathrm{~ms}^{-1} \mathrm{~d}^{-1}$. The star present signs of activity, we therefore corrected the RV using the RV-BVS correlation using Melo et al. (2007) method (see Appendix B). The trend is still visible with a lower slope $0.23 \mathrm{~ms}^{-1} \mathrm{~d}^{-1}$. We present the RV and corrected RV of HD 186704A in Fig. 3. The difference of one order of magnitude on the slope of the trend between Nidever et al. (2002) observations and ours can be explained by the fact that our observations were made when the SB was closer to the periastron or apoastron of its orbit.

\subsubsection{HD 181321}

We confirm that HD 181321 is an SB. Nordström et al. (2004) report a variation of $2.3 \mathrm{~km} \mathrm{~s}^{-1}$ over $9 \mathrm{yr}$, Guenther \& Esposito (2007) report a trend with a slope of $1.4 \mathrm{~km} \mathrm{~s}^{-1} \mathrm{yr}^{-1}$. Our observations spread on 3757 days (10.3 yr) cover at least two orbital periods. The star is active and show BVS variations with a period of 2-2.5 days that should correspond to the rotational period of the star. We use yorbit to fit the RV with two Keplerian models, one to fit the stellar activity variation and an another to fit the binary variations. We find two possible solutions for the companion, one with a period of 1600 days $(2.7 \mathrm{au})$ and a minimum mass of $0.1 M_{\odot}$, and a second with a period of 3200 days (4.4 au) 

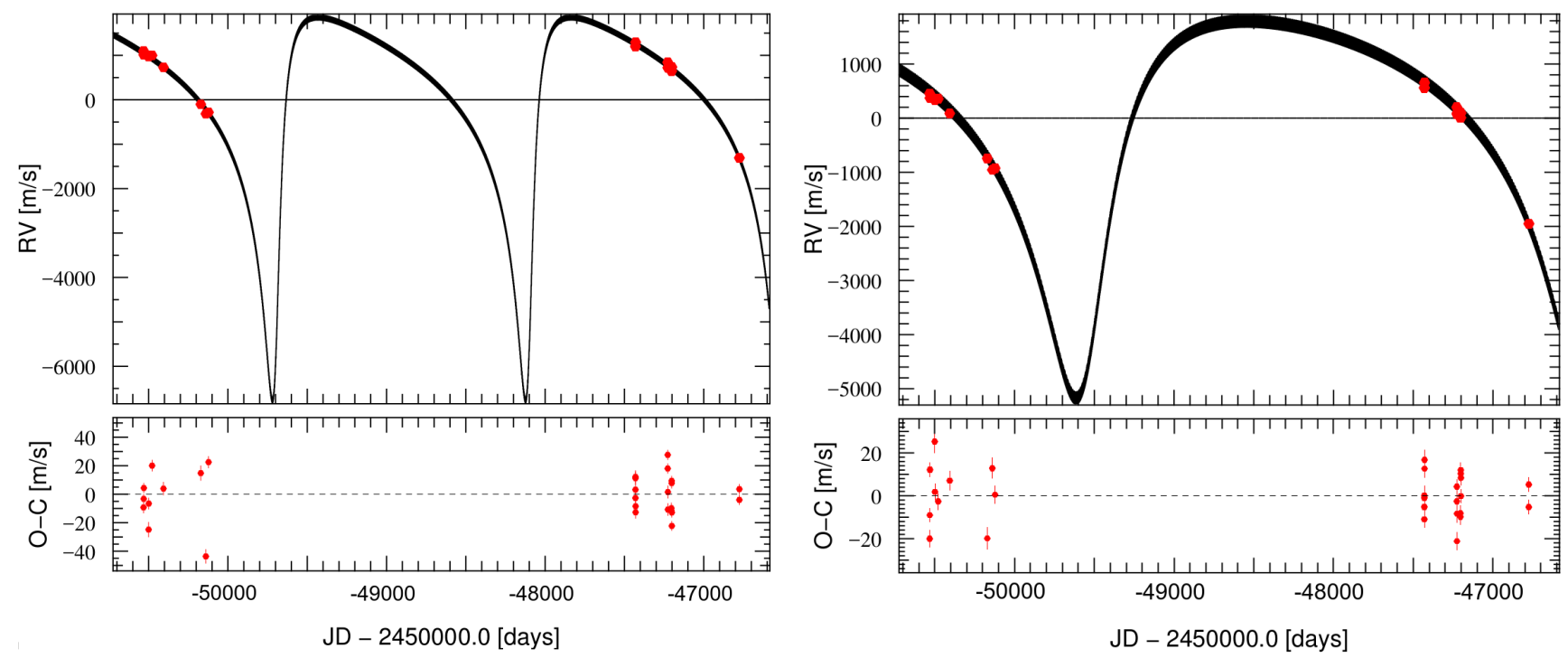

Fig. 4. Solutions of HD 181321 RV fit by the sum of two Keplerian (top) and their residuals (bottom). Left: 1600 days solution. Right: 3200 days solution.

and a minimum mass of $0.18 M_{\odot}$. In both cases, the eccentricity is $\sim 0.5$. We present those two solutions in Fig. 4. More data are needed in order to distinguish between those two solutions.

\subsubsection{HD 206893}

HD 206893 is a F5V star that hosts a directly imaged BD companion at a separation of 270 mas (Milli et al. 2017; Delorme et al. 2017). We recently reported in Grandjean et al. (2019) a long-term trend in the star RV coupled with pulsations with periods slightly less than one day. We performed an MCMC on both the RV trend, imaging data, and HIPPARCOS-Gaia proper motion measurements to constrain the orbit and dynamical mass of the $\mathrm{BD}$. We concluded that the trend can not be attributed to the BD as it leads to dynamical masses incompatible with the object's spectra. The presence of an inner companion that contributes significantly to the RV trend is suggested with a mass of $15 M_{\text {Jup }}$, and a period between 1.6 and $4 \mathrm{yr}$.

\subsubsection{HD 217987}

HD 217987 is a M2V, high proper motion star and exhibits a long-term RV trend induced by its secular acceleration. We therefore corrected the RV from the star secular acceleration in its proper motion we deduced from its paralax and proper motion reported in Gaia Collaboration (2018) (see Fig. 5). The corrected RV presents short period variations. The RV and BVS are correlated which indicate that the signal might be dominated by spots or plages (see Fig. 5). We observe a long-term variation in $\log R_{\mathrm{HK}}^{\prime}$, FWHM, and RV which are better fitted by a second degree polynomial model than with a linear model (see Fig. 5). This variation could be then attributed to a magnetic cycle of the star with a period greater than 5000 days. An analysis on a large number of $\mathrm{M}$ dwarf was made by Mignon et al. (in prep.), including this star and we should expect an offset of approximately $-10 \mathrm{~ms}^{-1}$ in the RV due to the HARPS fiber change of the 15 th of June 2015 for this star. We observe an offset in the star's BVS at this date date (see Fig. 5), but no significant offset in the RV. We use one template on all the data (see Sect. 2.3), the impact of the HARPS fiber change is then averaged. This can explain why we do not see a significant RV offset for this star.

\subsubsection{HIP 36985}

HIP 36985 is a M2V-type star reported as a wide binary with the system GJ 282AB at a separation of 1.09 (Poveda et al. 2009). We observe an RV long-term variation with an amplitude of $700 \mathrm{~ms}^{-1}$ on a time baseline of 1400 days in addition to a short-term variation with an amplitude of $50 \mathrm{~ms}^{-1}$. No correlation between RV and BVS is seen, which excludes stellar activity or pulsations as the origin of the long-term variations. The mean $\log R_{\mathrm{HK}}^{\prime}$ of our spectra of -4.3 indicates that the shortterm variations come from magnetic activity (spots). We fit the RV with yorbit using two Keplerian models, one to fit the longterm variations and an another to fit the companion variations. We present our best solution in Fig. 6 and the corresponding companion parameters in Table 1 . The companion minimum mass deduced from the present data is $29 M_{\text {Jup }}$ and the period is 8400 days ( $23 \mathrm{yr}$ ), corresponding to a semi-major axis of $7 \mathrm{au}$ and a projected separation of 497 mas. We observed HIP 36985 with SPHERE in 2018 and confirm the presence of a however lowmass star companion (Biller et al., in prep.). We find a period of 22 days for the short-term variations, while the rotation period is of $12 \pm 0.1$ days (Díez Alonso et al. 2019). The poor sampling of those short-term variations probably explains the strong difference with the rotation period.

\subsection{Giant planets}

No giant planet companion of period less than 1000 days is detected. In addition to the stars presented in Sect. 3, some stars presented RV variations without a significant correlation between RV and BVS. For these stars we compared the RV periodogram with the BVS periodogram and the time window periodogram. In all cases, putative periods associated with the RV were also present in the BVS or time window periodograms, or in both. This implies that these RV variations are most likely due to stellar activity or pulsations. We present as an example BD+20 2465 in Fig. 7. This star shows RV variations with 


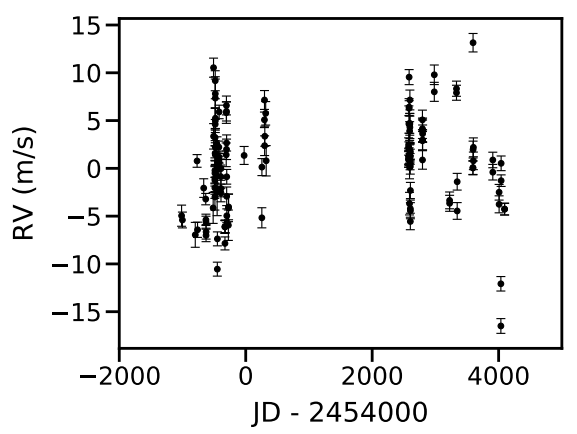

(a)

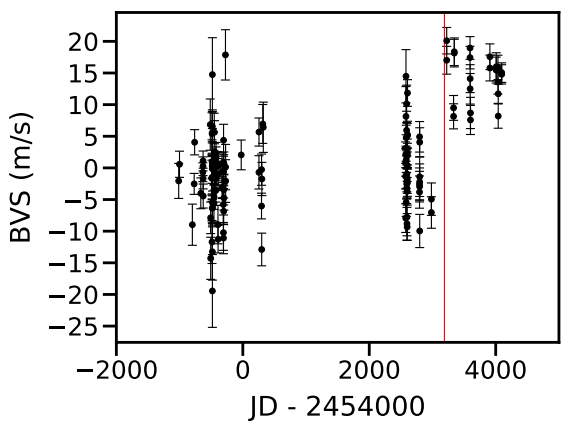

(b)

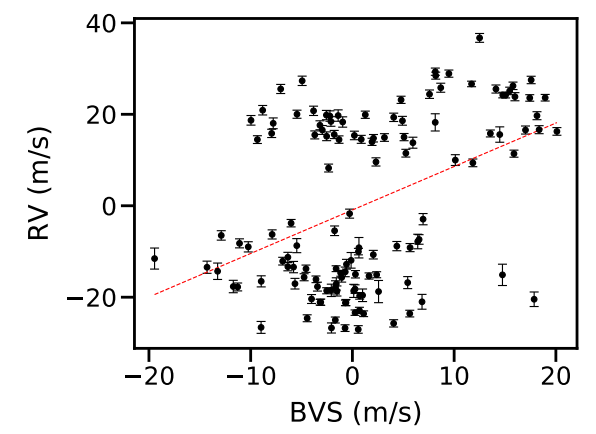

(c)

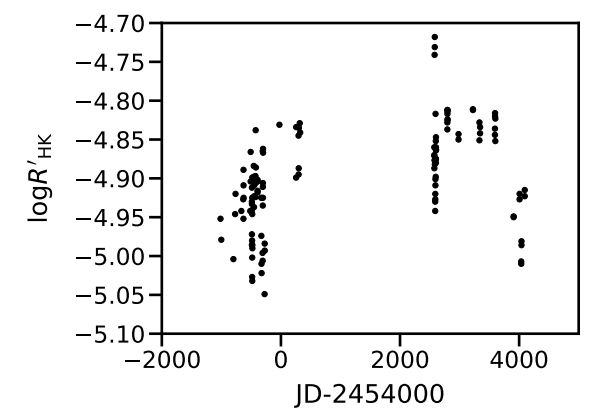

(d)

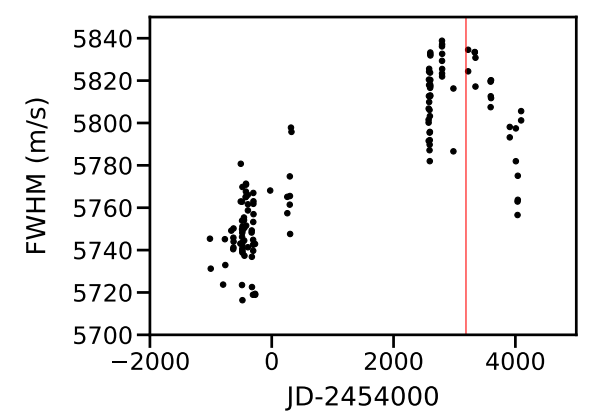

(e)

Fig. 5. HD 217987 summary. (a) RV time variations corrected from the secular acceleration drift. (b) BVS time variations, HARPS fiber change is shown with a vertical red line. (c) RV corrected from the secular acceleration drift vs BVS. The best linear fit is presentend in red dashed line. (d) $\log R_{\mathrm{HK}}^{\prime}$ time variations. (e) FWHM time variations.

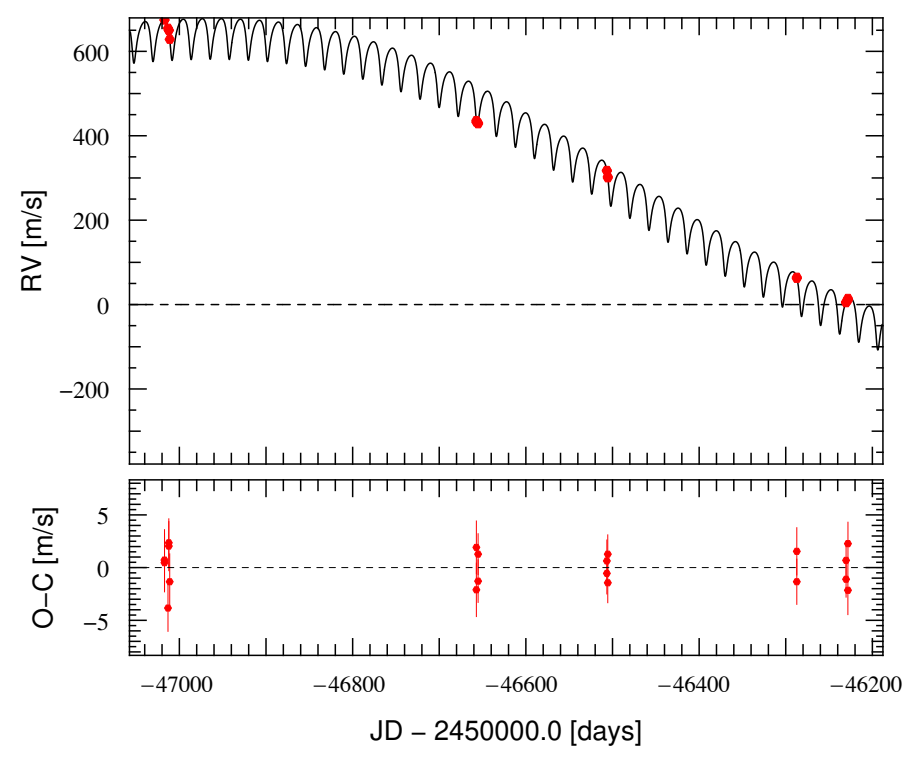

Fig. 6. Solution of HIP 36985 RV's fit by a sum of two keplerians and its residuals

periods near 2 days which are both present in the time window periodogram and BVS periodogram.

\section{Analysis}

\subsection{Stellar intrinsic variability}

Figure 8 displays the mean RV uncertainty vs. $B-V, v \sin i$ and $M_{*}$. We also display the RV rms vs. $B-V$, and age in Fig. 9. We observe that the mean RV uncertainty is correlated to the $v \sin i$
Table 1. HIP 36985B's orbital parameters.

\begin{tabular}{cc}
\hline \hline Parameters & Value \\
\hline$P$ (days) & $8500_{-1900}^{+1900}$ \\
$a(\mathrm{au})$ & $7_{-1.1}^{+1}$ \\
Separation (mas) & $500_{-80}^{+70}$ \\
$e$ & $0.55 \pm 0.04$ \\
$\omega\left(^{\circ}\right)$ & $50 \pm 67$ \\
$K\left(\mathrm{~ms}^{-1}\right)$ & $480 \pm 230$ \\
$M \sin i\left(M_{\text {Jup }}\right)$ & $29_{-16}^{+18}$ \\
\hline
\end{tabular}

(Pearson $\left.=-0.65, p_{\text {value }} \ll 1 \%\right)$. This is consistent with what is observed on older, AF main sequence stars by Borgniet et al. (2017, 2019). We observe a strong jitter for most of the stars. The ratio between RV rms and the mean RV uncertainty is between 390 and 1 with a median at 16.

The median RV rms is $49 \mathrm{~ms}^{-1}$ ( $300 \mathrm{~ms}^{-1}$ on average). This jitter is mainly caused by pulsations for early type stars (from A to F5V), and by spots and faculae for late type stars (>F5V). Those two regimes can be distinguished, as stars with pulsations shows a vertical spread of BVS(RV) diagram, whereas stars with spots present a correlation between RV and BVS (Lagrange et al. 2009a). The main origin of RV jitter is reported in Table A.2 for each target.

78 stars out of 89 of our sample present variations in their $\mathrm{Ca}$ lines. The median $\log R_{\mathrm{HK}}^{\prime}$ of our sample is -4.3 with a standard deviation of 0.2. 4 stars present signs of low activity $\left(\log R_{\mathrm{HK}}^{\prime}<-4.75\right), 59$ are active $\left(-4.75<\log R_{\mathrm{HK}}^{\prime}<-4.2\right)$ and 15 stars present signs of high activity $\left(\log R_{\mathrm{HK}}^{\prime}>-4.2\right)$. We 

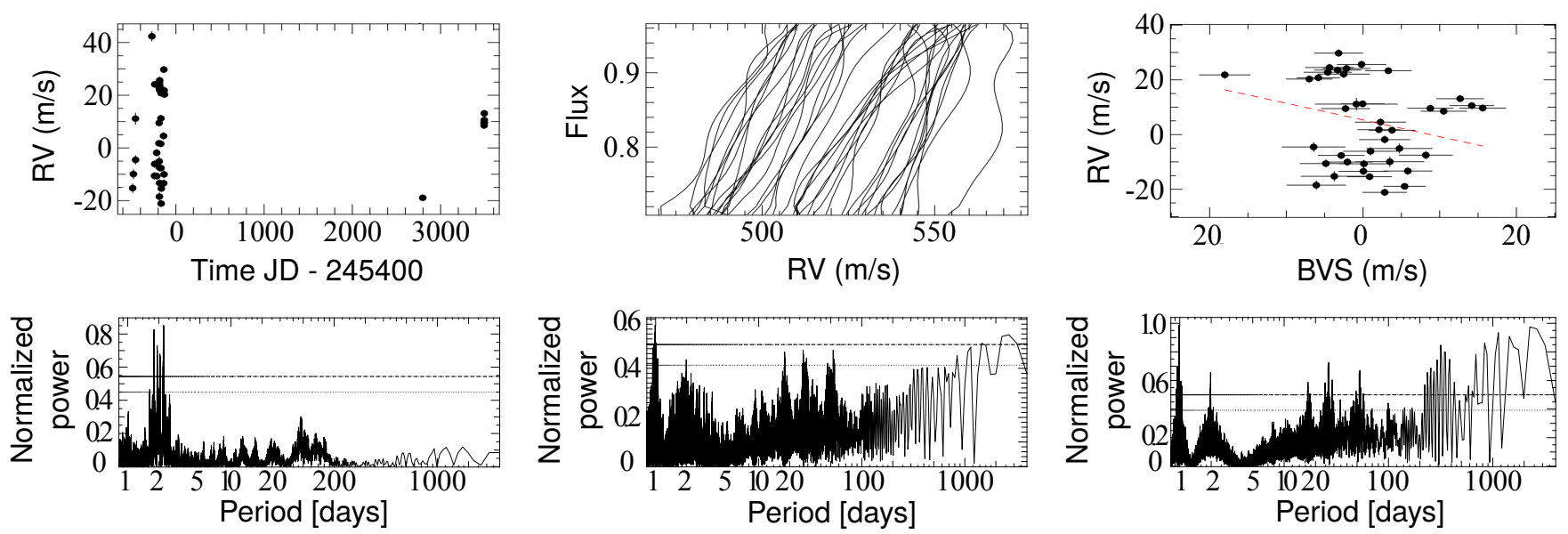

Fig. 7. BD+20 2465 summary. Top row: RV time variations, left: bisectors, middle: RV vs BVS (right). Bottom row: RV periodogram (left), BVS periodogram (middle), time window peridogram (right). The FAP at 1 and 10\% are presented respectively in dashed lines and in dotted lines.
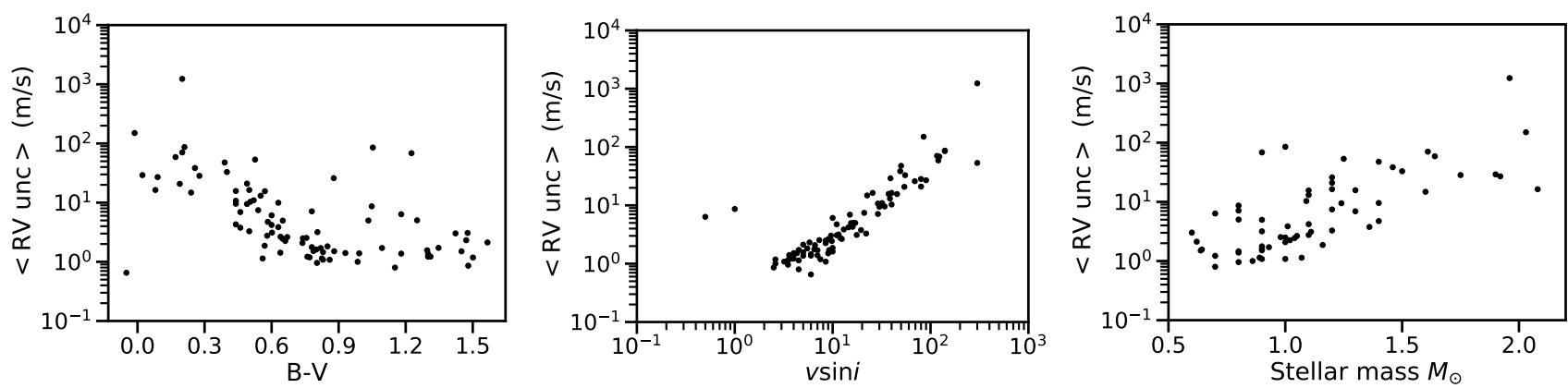

Fig. 8. Summary of the survey RV uncertainties. Mean RV uncertainty (accounting for the photon noise only) vs. $B-V$ (left), vs. $v \sin i$ (middle) and vs. $M_{\star}\left(\right.$ in $\left.M_{\odot}, r i g h t\right)$.
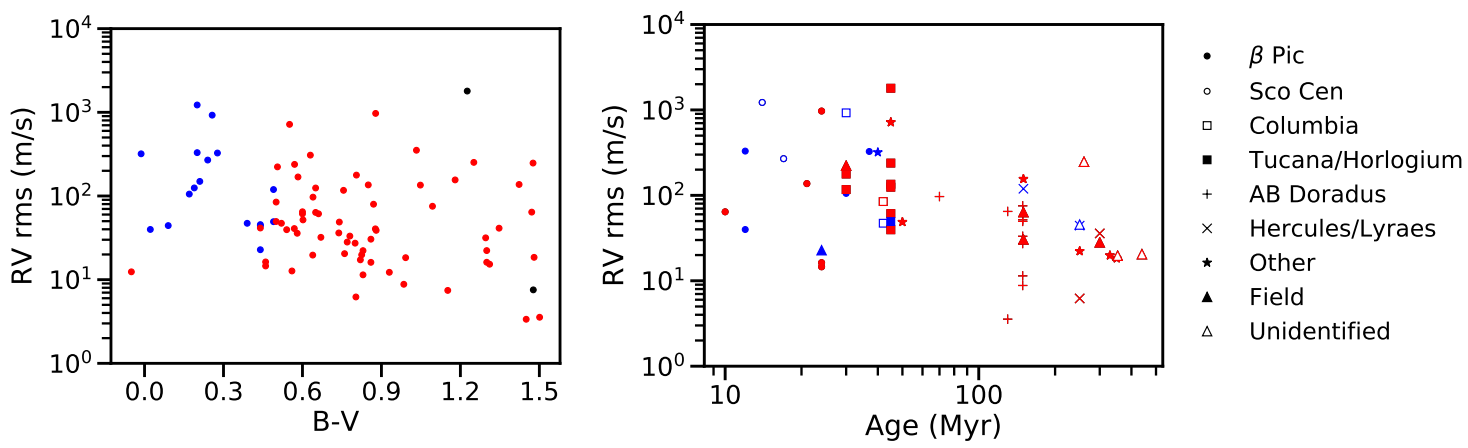

Fig. 9. Survey RV rms summary. Left: RV rms vs. $B-V$, right: RV rms and vs. age. Pulsating stars are plotted in blue, stars with RV dominated magnetic activity (spots) are plotted in red and stars with undetermined main source of RV are plotted in black. Stars with SB signature are not displayed (HD 106906, HD 131399, HD 177171). HD 197890 is not considered due to a too small set of data available.

present in Fig. $10 \log R_{\mathrm{HK}}^{\prime}$ vs. $B-V$. Some early F-type pulsating stars also present signs of actity when all late type stars present signs of activity.

\subsection{HARPS fiber change}

In June 2015, the fiber of the HARPS instrument was changed in order to increase its stability (Lo Curto et al. 2015). Lo Curto et al. (2015) show that it leads to a change in the instrument profile which impacts the CCF computation and therefore the RV and BVS computation. They observed an offset in the RV between the datasets taken before and after this change of the order of $15 \mathrm{~ms}^{-1}$ for old $\mathrm{F}$ to K-type stars, based on 19 stars
(Lo Curto et al. 2015). A more detailed analysis is currently underway for a large sample of close M-type main sequence stars (Mignon et al., in prep.). In this analysis, two reference spectra are computed, one before the change and one after. Our current set of spectra for each star is not big enough to build reference spectra as done in Mignon et al. (in prep.). The offset is different from one star to the other and its correlation with stellar parameters is not yet determined (spectral type, $v \sin i$ etc.). This offset has not been estimated on young stars before and the impact of high $v \sin i$ and strong jitter is not known. One should be careful before trying to correct this offset in order to not remove signal.

The difference between the mean of the RV before and after the fiber change can be measured, however, it won't probe the 


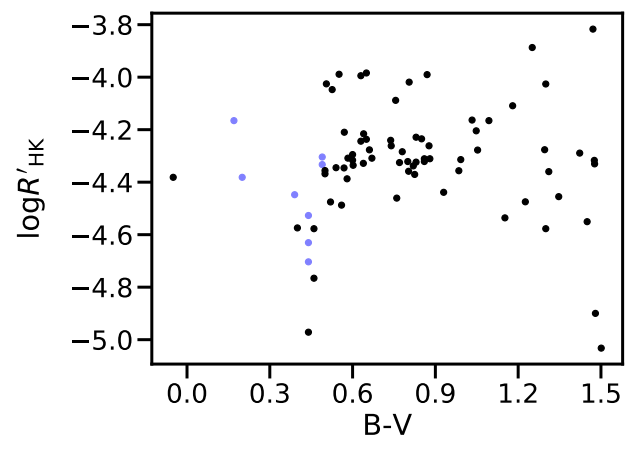

Fig. 10. Survey $\log R_{\mathrm{HK}}^{\prime}$ vs. $B-V$. Pulsating stars are plotted in blue.

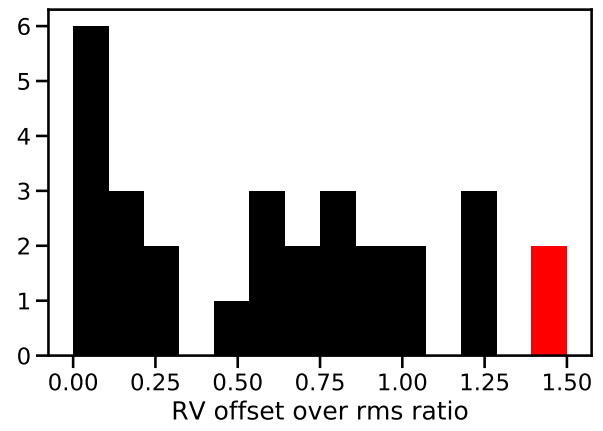

Fig. 11. Histogram of the ratio between the difference in the RV mean between before and after HARPS' fiber change, and the maximum of the rms before and after the fiber change. The stars that present a ratio greater than 1.3 are plotted in red.

offset alone, as long-term variations (star magnetic cycle, unseen companions, etc.) or a bad sampling of the jitter can also induce a difference in the mean of the RV.

We then decided to correct the offset only when it is significant enough. We compared the difference between the RV mean before and after the fiber change to the maximum of the rms before and after the fiber change to determine it. First, we selected the stars for which computing rms before and after the fiber change is relevant: we excluded the stars for which we have less than 10 spectra, less than 6 spectra before the fiber change, and less than 6 spectra after. We excluded the stars identified as SB (marked "B" in Table A.2) or presenting a long-term variation due to a companion (marked "T" in Table A.2). We also excluded the stars for which the RV amplitude is more than $900 \mathrm{~ms}^{-1}$ as the offset should be negligible compared to the jitter. We finally excluded HD 169178 that presents a trend due to a magnetic cycle. Finally, 29 stars were not excluded. We present the histogram of the ratio between the difference in mean of RV between before and after the fiber change and the maximum of the rms before and after the fiber change in Fig. 11. We chose to correct the RV offset for the stars that present a ratio larger than 1.3 a threshold that ensure a significant offset. Two stars correspond to this criterion. We present the characteristics of their offset in Table 2, and we present the correction of the offset for one of them in Fig. B.2. They have G to K-types and present an offset of the order of $50 \mathrm{~ms}^{-1}$. Such offsets are three times larger than those founds on old stars (Lo Curto et al. 2015).

\subsection{Exclusion of peculiar stars and RV correction for further analysis}

In order to better estimate the number of potentially missed planets in our survey, we excluded some stars from our analyze
Table 2. Parameters of the stars that present a significant offset.

\begin{tabular}{lccccc}
\hline \hline Name & ST & $B-V$ & $\begin{array}{c}\langle F W H M\rangle \\
\left(\mathrm{km} \mathrm{s}^{-1}\right)\end{array}$ & $\begin{array}{c}\text { Offset } \\
\left(\mathrm{ms}^{-1}\right)\end{array}$ & Ratio \\
\hline HD 7661 & K0V & 0.77 & 9.2 & 42 & 1.4 \\
HD 218860 & G8V & 0.74 & 11.7 & 57 & 1.4 \\
\hline
\end{tabular}

Notes. The offset is the difference between the RV mean before and after HARPS' fiber change, and the ratio is the ratio between the offset and the maximum of the rms before and after the fiber change.

and made some corrections on others before computing the detection limits. We excluded SB stars (HD 106906, HD 131399 , HD 177171, HD 181321). Further, we excluded HD 116434 since its high value of $v \sin i\left(>200 \mathrm{~km} \mathrm{~s}^{-1}\right)$ prevents the measurements of BVS. We also excluded HD 197890 for which our data are too sparse to reliably quantify the detection limits ( 3 spectra). Thus leads to 83 stars, 23 A-F stars, 52 F-K stars, and 8 K-M stars.

For stars with RV dominated by spots (marked $\mathrm{A}$ in the Table A.2) we corrected their RV from the RV-BVS correlation using Melo et al. (2007) method (see Appendix B). We corrected HD 217987 RV from its proper motion and HD 186704 RV from its long-term trend with a linear regression. For $\beta$ Pic, we considered the RV corrected from its pulsations as well as $\beta$ Pic $\mathrm{b}$ and c contributions (Lagrange et al. 2019b).

For the stars for which we identified a non ambiguous offset in the RV, we corrected their RV from this offset (see Table 2).

\subsection{Detection limits}

We compute $m_{p} \sin i$ detection limits for periods between 1 and 1000 days in the GP domain (between 1 and $13 M_{\text {Jup }}$ ), and in the BD domain (between 13 and $80 M_{\mathrm{Jup}}$ ). We use the Local Power Analysis (LPA; Meunier et al. 2012; Borgniet et al. 2017) which determines, for all periods $P$, the minimum $m_{p} \sin i$ for which a companion on a circular orbit with a period $P$ would lead to a signal consistent with the data, by comparing the synthetic companion maximum power of its periodogram to the maximum power of the data periodogram within a small period range around the period $P$.

We then compute the completeness function $C\left(m_{p} \sin i, P\right)$ which corresponds for a given couple $\left(m_{p} \sin i, P\right)$ to the fraction of stars for which the companion is excluded by the detection limits (Borgniet et al. 2017). We present the 40-80\% search completeness in Fig. 12.

\subsection{Companion occurrence rates}

We compute the upper limits of companions occurrence rates for our 83 stars in the GP $\left(1-13 M_{\text {Jup }}\right)$ and BD $\left(13-80 M_{\text {Jup }}\right)$ domains for $\mathrm{AF}(B-V \in[-0.05: 0.52])$, FK $(B-V \in[0.52$ : $1.33]), \mathrm{KM}(B-V \geq 1.33)$ type stars for different ranges of periods: $1-10,10-100,100-1000$, and 1-1000 days. We use the method described in Borgniet et al. (2017) to compute the occurrence rates and to correct them from the estimated number of missed companions $n_{\text {miss }}$ derived from the search completeness.

We present the upper limits of the occurrence rates for all stars in Fig. 13 and for AF, FK, M, and all stars in Table 3. The GP occurrence rate is below $2_{-2}^{+3} \%(1 \sigma)$ and the BD occurrence rate is below $1_{-1}^{+3} \%(1 \sigma)$ for periods under 1000 days. 
Table 3. GP $\left(m_{p} \sin i \in[1,13] M_{\mathrm{Jup}}\right)$ and $\mathrm{BD}\left(m_{p} \sin i \in[13,80] M_{\mathrm{Jup}}\right)$ occurrence rates around young nearby stars.

\begin{tabular}{|c|c|c|c|c|c|c|c|c|}
\hline \multirow{2}{*}{$\begin{array}{l}m_{p} \sin i \\
\text { interval } \\
\left(M_{\text {Jup }}\right)\end{array}$} & \multirow{2}{*}{$\begin{array}{l}\text { Orbital period } \\
\text { interval } \\
\text { (day) }\end{array}$} & \multirow[t]{2}{*}{$B-V$} & \multirow{2}{*}{$\begin{array}{c}\text { Search } \\
\text { completeness } \\
C(\%)\end{array}$} & \multirow{2}{*}{$\begin{array}{l}\text { Detected } \\
\text { GP systems }\end{array}$} & \multirow{2}{*}{$\begin{array}{c}\text { Missed } \\
\text { GP systems } \\
\text { upper limit }\end{array}$} & \multirow{2}{*}{$\begin{array}{l}\text { GP occurrence rate } \\
\text { upper limit } \\
(\%)\end{array}$} & \multicolumn{2}{|c|}{ Confidence intervals } \\
\hline & & & & & & & $\begin{array}{l}\sigma \\
(\%)\end{array}$ & $\begin{array}{l}2 \sigma \\
(\%)\end{array}$ \\
\hline \multirow{12}{*}{$\begin{array}{l}1-13 \\
\text { (GP) }\end{array}$} & \multirow{4}{*}{$1-10$} & All & 94 & 0 & 0.1 & 1.3 & $0-4.1$ & $0-7.0$ \\
\hline & & {$[-0.05: 0.52]$} & 89 & $\mathbf{0}$ & 0.1 & 4.9 & 0-14.7 & $0-24.0$ \\
\hline & & {$[0.52: 1.33]$} & 95 & 0 & 0.1 & 2.0 & $0-6.4$ & $0-10.8$ \\
\hline & & $\geq 1.33$ & 98 & 0 & 0.0 & 12.8 & $0-32.9$ & $0-50.0$ \\
\hline & \multirow[t]{4}{*}{$1-100$} & All & 89 & 0 & 0.1 & 1.4 & $0-4.4$ & $0-7.4$ \\
\hline & & {$[-0.05: 0.52]$} & 81 & 0 & 0.2 & 5.4 & $0-16.3$ & 0-26.7 \\
\hline & & {$[0.52: 1.33]$} & 91 & 0 & 0.1 & 2.1 & $0-6.7$ & $0-11.3$ \\
\hline & & $\geq 1.33$ & 92 & 0.1 & 0.0 & 13.6 & $0-35.0$ & $0-53.1$ \\
\hline & \multirow{4}{*}{$1-1000$} & All & 80 & 0 & 0.3 & 1.5 & $0-4.9$ & $0-8.3$ \\
\hline & & {$[-0.05: 0.52]$} & 71 & $\mathbf{0}$ & 0.4 & 6.2 & 0-18.6 & $0-30.5$ \\
\hline & & {$[0.52: 1.33]$} & 82 & 0 & 0.2 & 2.4 & $0-7.5$ & $0-12.6$ \\
\hline & & $\geq 1.33$ & 86 & 0 & 0.2 & 14.5 & $0-37.4$ & $0-56.6$ \\
\hline \multirow{12}{*}{$\begin{array}{l}13-80 \\
\text { (BD) }\end{array}$} & \multirow[t]{4}{*}{$1-10$} & All & 99 & 0 & 0.0 & 1.2 & $0-3.9$ & $0-6.6$ \\
\hline & & {$[-0.05: 0.52]$} & 99 & $\mathbf{0}$ & 0.0 & 4.4 & $0-13.2$ & $0-21.6$ \\
\hline & & {$[0.52: 1.33]$} & 99 & 0 & 0.0 & 1.9 & $0-6.2$ & $0-10.4$ \\
\hline & & $\geq 1.33$ & 99 & 0 & 0.0 & 12.6 & $0-32.6$ & $0-49.5$ \\
\hline & \multirow[t]{4}{*}{$1-100$} & All & 97 & 0 & 0.0 & 1.2 & $0-4.0$ & $0-6.8$ \\
\hline & & {$[-0.05: 0.52]$} & 95 & $\mathbf{0}$ & 0.0 & 4.6 & 0-13.7 & $0-22.5$ \\
\hline & & {$[0.52: 1.33]$} & 97 & 0 & 0.0 & 2.0 & $0-6.3$ & $0-10.6$ \\
\hline & & $\geq 1.33$ & 93 & 0 & 0.1 & 13.4 & $0-34.7$ & $0-52.6$ \\
\hline & \multirow[t]{4}{*}{$1-1000$} & All & 92 & 0 & 0.1 & 1.3 & $0-4.2$ & $0-7.2$ \\
\hline & & {$[-0.05: 0.52]$} & 89 & 0 & 0.1 & 4.9 & 0-14.7 & $0-24.0$ \\
\hline & & {$[0.52: 1.33]$} & 93 & 0 & 0.1 & 2.1 & $0-6.6$ & $0-11.1$ \\
\hline & & $\geq 1.33$ & 90 & 0 & 0.1 & 13.8 & $0-35.7$ & $0-54.1$ \\
\hline
\end{tabular}

Notes. The parameters are displayed in normal, bold, italic or bold and italic fonts when considering the full star sample, the early type AF stars, FK-type stars or KM-type stars, respectively.

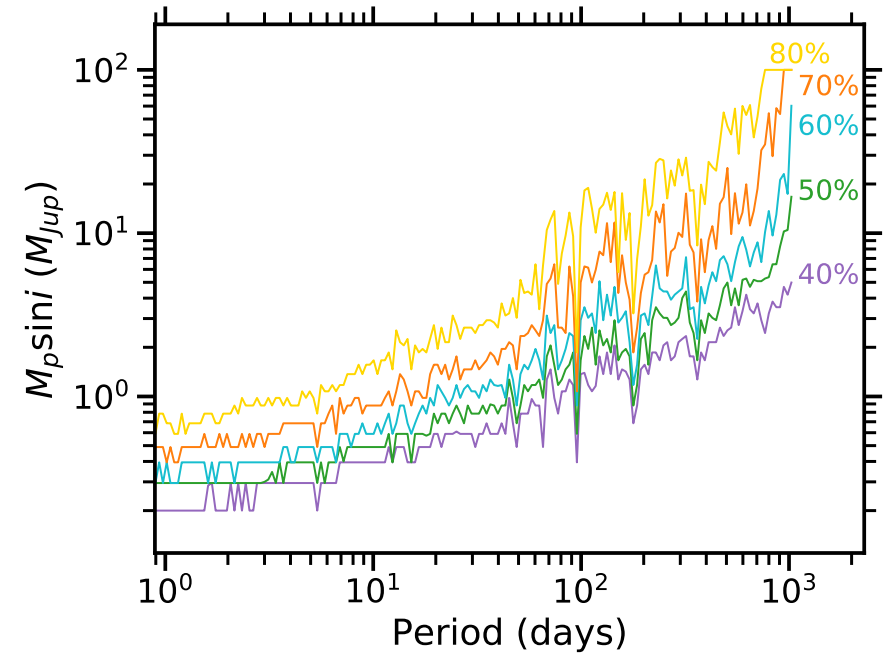

Fig. 12. Search completeness of our survey, corresponding to the lower $m_{p} \sin i$ for which $X \%$ of the star of the survey have detection limits below this $m_{p} \sin i$ at a given period $P$. From bottom to top $40-80 \%$.

\subsection{Comparaison to surveys on main sequence stars}

No GP companion with periods lower than 1000 days was detected in the present survey. This non-detection is robust

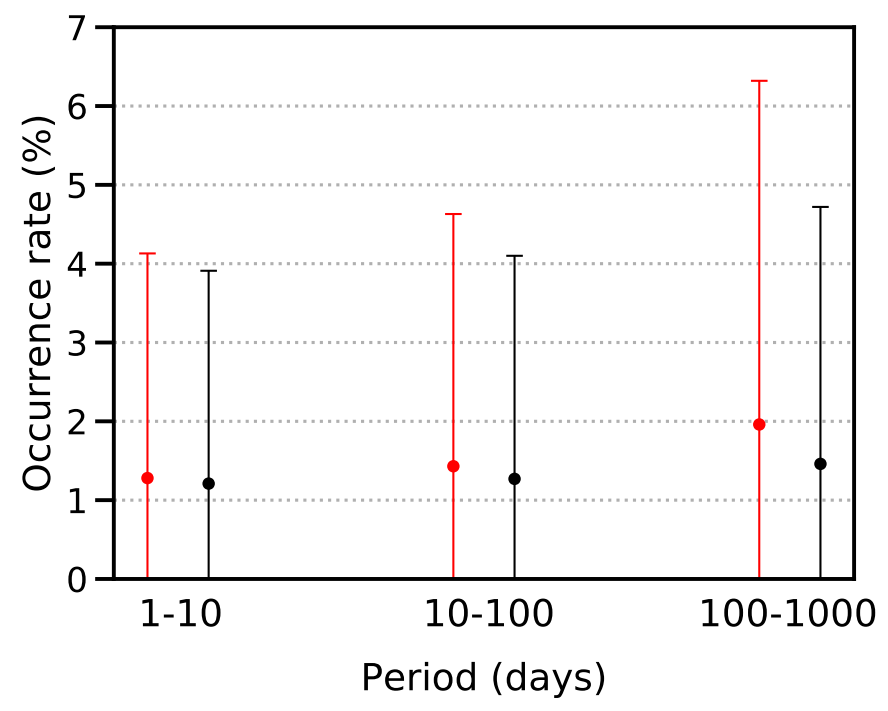

Fig. 13. Upper limits $(1 \sigma)$ on the occurrence rates for our survey for period ranges of $1-10,10-100$, and 100-1000 days in the GP domain (1-13 $M_{\text {Jup }}$, red) and BD domain (13-80 $M_{\text {Jup }}$, black).

for $\mathrm{HJ}$ as $70 \%$ of the star of the survey have detection limits lower than $1 M_{\text {Jup }}$ for period lower than 10 days. The completeness of the survey is over $71 \%$ for AF and FK stars and 
$n_{\text {miss }}<0.4$ for AF and FK stars in the 1-1000 days domain (cf. Table 3). However, we may have missed some planets with low masses and long period as only $40 \%$ of the stars of the survey have detection limits lower than $5 M_{\text {Jup }}$ between 100 and 1000 days.

We statistically tested if our GP non-detection implies GP occurrence rates around young stars significantly lower than around older main sequence (MS) stars, using the $p$-value formalism. The $p$-value is the probability to get the observed results given a null hypothesis, if it is lower than $10 \%$ then the null hypothesis can be rejected. We tested the following null hypothesis: GP occurrence rates are identical around young and main sequence stars in the same mass and period range.

We first applied this formalism in the 1-1000 days domain with a survey that have a completeness similar to ours. For AF MS stars the GP occurrence rate in this domain was estimated at $2.5_{-0.5}^{+2.5 \%}$ (Borgniet et al. 2019). We detected 0 companion out of 23 stars, the corresponding $p$-value is $56_{-21}^{+7} \%$. The null hypothesis can not be rejected.

Then, we applied this formalism in the HJ ( $P<10$ days) domain. For FK MS stars, the occurrence rate for $\mathrm{HJ}$ in this domain was estimated at: $0.46_{-0.3}^{+0.3} \%$ by Cumming et al. (2008) and at $1.2 \pm 0.38 \%$ by Wright et al. (2012). We detected 0 companion out of 52 stars, the corresponding $p$-value are respectively $79_{-11}^{+13} \%$ and $53_{-9}^{+12} \%$. The null hypothesis can not be rejected.

There is no evidence for a difference in occurrence rates of $\mathrm{HJ}$ between young and MS stars.

BD companions occurrence rates around MS stars is low: $f \leq 1.1_{-1.1}^{+4.1} \%$ for periods less than 1000 days (Borgniet et al. 2017), our non detection is not surprising. A bigger sample is needed to determine if BD occurrence rates around young and MS stars are different or not.

\section{Conclusions}

We observed 89 young A- to M-type stars over 3 yr or more with HARPS to search for GP and BD with periods less than 1000 days. This survey allowed to detect close binaries around HD 106906 and around HD 131399 (Lagrange et al. 2015, 2017). We constrained the period of HD 181321B and confirmed the RV trend in HD 186704's RV. We also discovered a low-mass star companion to HIP 36985 . No GP companion was detected in this survey. We obtain upper limits on the GP and BD occurrence rates, they are respectively $2_{-2}^{+3} \%$ and $1_{-1}^{+3} \%$ for periods of less than 1000 days. Our comparison of these occurrence rates to those derived for MS stars (Borgniet et al. 2019; Cumming et al. 2008; Wright et al. 2012) indicates that there is no evidence for a difference in occurrence rates of $\mathrm{HJ}$ between young and MS stars.

The forthcoming analysis of our SOPHIE survey around young stars and of our on-going HARPS survey on Sco-Cen stars will add 60 and 80 stars, respectively, in our analysis. This will allow for the derivation of more accurate occurrence rates and it will help in the search for the possible impact of system ages on occurrence rates.

Acknowledgements. We acknowledge support from the French CNRS and from the Agence Nationale de la Recherche (ANR grant GIPSE ANR-14-CE330018). This work has been supported by a grant from Labex OSUG@2020 (Investissements d'avenir - ANR10 LABX56). These results have made use of the SIMBAD database, operated at the CDS, Strasbourg, France. ESO SD acknowledges the support by INAF/Frontiera through the "Progetti Premiali" funding scheme of the Italian Ministry of Education, University, and Research.

\section{References}

Ammler-von Eiff, M., \& Guenther, E. W. 2009, A\&A, 508, 677 Aumann, H. H. 1985, PASP, 97, 885

Backman, D. E., \& Gillett, F. C. 1987, in Cool Stars, Stellar Systems and the Sun, Lecture Notes in Physics, eds. J. L. Linsky, \& R. E. Stencel (Berlin: Springer Verlag), 291, 340

Bailey, V., Meshkat, T., Reiter, M., et al. 2014, ApJ, 780, L4

Beichman, C. A., Bryden, G., Stapelfeldt, K. R., et al. 2006, ApJ, 652, 1674

Beust, H., Lagrange-Henri, A. M., Vidal-Madjar, A., \& Ferlet, R. 1989, A\&A, 223, 304

Bonavita, M., Desidera, S., Thalmann, C., et al. 2016, A\&A, 593, A38

Borgniet, S., Lagrange, A. M., Meunier, N., \& Galland, F. 2017, A\&A, 599, A57

Borgniet, S., Lagrange, A.-M., Meunier, N., et al. 2019, A\&A, 621, A87

Bouchy, F., \& Sophie Team. 2006, in Tenth Anniversary of 51 Peg-b: Status of and Prospects for Hot Jupiter Studies, eds. L. Arnold, F. Bouchy, \& C. Moutou (Paris: Frontier Group), 319

Brandt, T. D., Kuzuhara, M., McElwain, M. W., et al. 2014, ApJ, 786, 1

Carpenter, J. M., Bouwman, J., Mamajek, E. E., et al. 2009, ApJS, 181, 197

Chauvin, G., Desidera, S., Lagrange, A.-M., et al. 2017a, in SF2A-2017: Proceedings of the Annual meeting of the French Society of Astronomy and Astrophysics, ed. C. Reylé, P. Di Matteo, F. Herpin, E. Lagadec, A. Lançon, Z. Meliani, \& F. Royer, 331

Chauvin, G., Desidera, S., Lagrange, A.-M., et al. 2017b, A\&A, 605, L9

Chen, C. H., Patten, B. M., Werner, M. W., et al. 2005, ApJ, 634, 1372

Chen, C. H., Mittal, T., Kuchner, M., et al. 2014, ApJS, 211, 25

Choquet, E., Perrin, M. D., Chen, C. H., et al. 2016, ApJ, 817, L2

Churcher, L., Wyatt, M., \& Smith, R. 2011, MNRAS, 410, 2

Cumming, A., Butler, R. P., Marcy, G. W., et al. 2008, PASP, 120, 531

de la Reza, R., \& Pinzón, G. 2004, ApJ, 128, 1812

Delorme, P., Lagrange, A. M., Chauvin, G., et al. 2012, A\&A, 539, A72

Delorme, P., Schmidt, T., Bonnefoy, M., et al. 2017, A\&A, 608, A79

Desidera, S., Covino, E., Messina, S., et al. 2015, A\&A, 573, A126

Desort, M., Lagrange, A. M., Galland, F., Udry, S., \& Mayor, M. 2007, A\&A, 473, 983

Díez Alonso, E., Caballero, J. A., Montes, D., et al. 2019, A\&A, 621, A126

Donaldson, J. K., Roberge, A., Chen, C. H., et al. 2012, ApJ, 753, 147

Donati, J. F., Moutou, C., Malo, L., et al. 2016, Nature, 534, 662

Esposito, M., Guenther, E., Hatzes, A. P., \& Hartmann, M. 2006, in Tenth Anniversary of 51 Peg-b: Status of and Prospects for Hot Jupiter Studies, eds. L. Arnold, F. Bouchy, \& C. Moutou (Paris: Frontier Group), 127

Figueira, P., Marmier, M., Bonfils, X., et al. 2010, A\&A, 513, L8

Folsom, C. P., Bouvier, J., Petit, P., et al. 2018, MNRAS, 474, 4956

Frankowski, A., \& Jorissen, A. 2007, Balt. Astron., 16, 104

Fuhrmann, K., Chini, R., Kaderhandt, L., \& Chen, Z. 2017, ApJ, 836, 139

Gaia Collaboration (Brown, A. G. A., et al.) 2018, A\&A, 616, A1

Galland, F., Lagrange, A. M., Udry, S., et al. 2005a, A\&A, 443, 337

Galland, F., Lagrange, A. M., Udry, S., et al. 2005b, A\&A, 444, L21

Gallet, F., \& Bouvier, J. 2015, A\&A, 577, A98

Golimowski, D. A., Krist, J. E., Stapelfeldt, K. R., et al. 2011, AJ, 142, 30

Grandjean, A., Lagrange, A. M., Beust, H., et al. 2019, A\&A, 627, L9

Guenther, E. W., \& Esposito, E. 2007, ArXiv e-prints [arXiv:astro-ph/0701293]

Hillenbrand, L. A., Carpenter, J. M., Kim, J. S., et al. 2008, ApJ, 677, 630

Hobbs, L. M., Vidal-Madjar, A., Ferlet, R., Albert, C. E., \& Gry, C. 1985, ApJ, 293, L29

Holland, W. S., Greaves, J. S., Zuckerman, B., et al. 1998, Nature, 392, 788

Huélamo, N., Figueira, P., Bonfils, X., et al. 2008, A\&A, 489, L9

Kalas, P., Liu, M. C., \& Matthews, B. C. 2004, Science, 303, 1990

Kiefer, F., Lecavelier des Etangs, A., Boissier, J., et al. 2014, Nature, 514, 462

Kiraga, M. 2012, Acta Astron., 62, 67

Kley, W., \& Nelson, R. P. 2012, ARA\&A, 50, 211

Koen, C., \& Eyer, L. 2002, MNRAS, 331, 45

Kovári, Z., Strassmeier, K., Granzer, T., et al. 2004, A\&A, 417, 1047

Lagrange-Henri, A. M., Vidal-Madjar, A., \& Ferlet, R. 1988, A\&A, 190, 275

Lagrange, A. M., Desort, M., Galland, F., Udry, S., \& Mayor, M. 2009a, A\&A, 495, 335

Lagrange, A. M., Gratadour, D., Chauvin, G., et al. 2009b, A\&A, 493, L21

Lagrange, A. M., De Bondt, K., Meunier, N., et al. 2012, A\&A, 542, A18

Lagrange, A.-M., Meunier, N., Chauvin, G., et al. 2013, A\&A, 559, A83

Lagrange, A.-M., Mathias, P., \& Absil, O. 2015, A\&A, submitted

Lagrange, A.-M., Keppler, M., Beust, H., et al. 2017, A\&A, 608, L9

Lagrange, A. M., Boccaletti, A., Langlois, M., et al. 2019a, A\&A, 621, L8

Lagrange, A. M., Meunier, N., Rubini, P., et al. 2019b, Nat. Astron., 421

Lawler, S. M., Beichman, C. A., Bryden, G., et al. 2009, ApJ, 705, 89

Lindgren, S., \& Heiter, U. 2017, A\&A, 604, A97 
Lo Curto, G., Pepe, F., Avila, G., et al. 2015, The Messenger, 162, 9 Mamajek, E. E. 2012, ApJ, 754, L20

Mamajek, E. E., Meyer, M. R., Hinz, P. M., et al. 2004, ApJ, 612, 496 Mannings, V., \& Barlow, M. J. 1998, ApJ, 497, 330

Mayor, M., Pepe, F., Queloz, D., et al. 2003, The Messenger, 114, 2 McDonald, I., Zijlstra, A. A., \& Boyer, M. L. 2012, MNRAS, 427, 343

Melo, C., Santos, N. C., Gieren, W., et al. 2007, A\&A, 467, 721

Meshkat, T., Bailey, V., Rameau, J., et al. 2013, ApJ, 775, L40

Messina, S., Desidera, S., Turatto, M., Lanzafame, A. C., \& Guinan, E. F. 2010 , A\&A, 520, A15

Messina, S., Millward, M., Buccino, A., et al. 2017, A\&A, 600, A83

Meunier, N., Lagrange, A. M., \& De Bondt, K. 2012, A\&A, 545, A87

Meyer, M. R., Hillenbrand, L. A., Backman, D. E., et al. 2004, ApJS, 154, 422

Meyer, M. R., Hillenbrand, L. A., Backman, D., et al. 2006, PASP, 118, 1690

Meyer, M. R., Carpenter, J. M., Mamajek, E. E., et al. 2008, ApJ, 673, L181

Milli, J., Hibon, P., Christiaens, V., et al. 2017, A\&A, 597, L2

Montesinos, B., Eiroa, C., Mora, A., \& Merín, B. 2009, A\&A, 495, 901

Montet, B. T., Crepp, J. R., Johnson, J. A., Howard, A. W., \& Marcy, G. W. 2014, ApJ, 781, 28

Moór, A., Ábrahám, P., Kóspál, A., et al. 2013, ApJ, 775, L51

Morales, F. Y., Bryden, G., Werner, M. W., \& Stapelfeldt, K. R. 2016, ApJ, 831,

Morin, J., Donati, J. F., Petit, P., et al. 2008, MNRAS, 390, 567

Moro-Martín, A., Marshall, J. P., Kennedy, G., et al. 2015, ApJ, 801, 143

Nidever, D. L., Marcy, G. W., Butler, R. P., Fischer, D. A., \& Vogt, S. S. 2002, ApJS, 141, 503

Nielsen, E. L., De Rosa, R. J., Rameau, J., et al. 2017, AJ, 154, 218

Nordström, B., Mayor, M., Andersen, J., et al. 2004, A\&A, 418, 989

Olmedo, M., Chávez, M., Bertone, E., \& De la Luz, V. 2013, PASP, 125, 1436

Passegger, V. M., Reiners, A., Jeffers, S. V., et al. 2018, A\&A, 615, A6

Patel, R. I., Metchev, S. A., \& Heinze, A. 2014, ApJS, 212, 10

Paulson, D. B., \& Yelda, S. 2006, PASP, 118, 706

Pecaut, M. J., Mamajek, E. E., \& Bubar, E. J. 2012, ApJ, 746, 154
Plavchan, P., Werner, M. W., Chen, C. H., et al. 2009, ApJ, 698, 1068 Pollack, J. B., Hubickyj, O., Bodenheimer, P., et al. 1996, Icarus, 124, 62 Poveda, A., Allen, C., Costero, R., Echevarría, J., \& Hernández-Alcántara, A. 2009, ApJ, 706, 343

Rameau, J., Chauvin, G., Lagrange, A.-M., et al. 2013a, ApJ, 772, L15

Rameau, J., Chauvin, G., Lagrange, A. M., et al. 2013b, A\&A, 553, A60 Rebull, L. M., Stapelfeldt, K. R., Werner, M. W., et al. 2008, ApJ, 681, 1484 Rebull, L. M., Stauffer, J. R., Bouvier, J., et al. 2016, AJ, 152, 114

Rhee, J. H., Song, I., Zuckerman, B., \& McElwain, M. 2007, ApJ, 660, 1556 Rodet, L., Beust, H., Bonnefoy, M., et al. 2017, A\&A, 602, A12

Schneider, G., Silverstone, M. D., Hines, D. C., et al. 2006, ApJ, 650, 414 Ségransan, D., Mayor, M., Udry, S., et al. 2011, A\&A, 535, A54 Sierchio, J. M., Rieke, G. H., Su, K. Y. L., \& Gáspár, A. 2014, ApJ, 785, 33 Simon, M., \& Schaefer, G. H. 2011, ApJ, 743, 158 Smith, B. A., \& Terrile, R. J. 1984, Science, 226, 1421

Soto, M. G., Jenkins, J. S., \& Jones, M. I. 2015, MNRAS, 451, 3131

Soummer, R., Perrin, M. D., Pueyo, L., et al. 2014, ApJ, 786, L23

Stauffer, J., Rebull, L., Bouvier, J., et al. 2016, AJ, 152, 115

Strassmeier, K. G., Pichler, T., Weber, M., \& Granzer, T. 2003, A\&A, 411, 595

Teyssandier, J., Lai, D., \& Vick, M. 2019, MNRAS, 486, 2265

Tokovinin, A. 2014, AJ, 147, 87

Tremko, J., Bakos, G. A., Žižňovský, J., \& Pribulla, T. 2010, Contrib. Astron. Observ. Skalnate Pleso, 40, 83

van Leeuwen, F. 2007, A\&A, 474, 653

Vigan, A., Bonavita, M., Biller, B., et al. 2017, A\&A, 603, A3

Wagner, K., Apai, D., Kasper, M., et al. 2016, Science, 353, 673

Weise, P., Launhardt, R., Setiawan, J., \& Henning, T. 2010, A\&A, 517, A88

Wright, N. J., Drake, J. J., Mamajek, E. E., \& Henry, G. W. 2011, ApJ, 743, 48 Wright, J. T., Marcy, G. W., Howard, A. W., et al. 2012, ApJ, 753, 160

Yu, L., Donati, J.-F., Hébrard, E. M., et al. 2017, MNRAS, 467, 1342 Zuckerman, B., \& Song, I. 2004, ApJ, 603, 738

Zuckerman, B., Rhee, J. H., Song, I., \& Bessell, M. S. 2011, ApJ, 732, 61

Zuckerman, B., Vican, L., Song, I., \& Schneider, A. 2013, ApJ, 778, 5 


\section{Appendix A: Sample}

Table A.1. Stars characteristics for the 89 stars of our HARPS RV survey.

\begin{tabular}{|c|c|c|c|c|c|c|c|c|}
\hline $\begin{array}{l}\text { Name } \\
\mathrm{HD} / \mathrm{BD} / \mathrm{CD}\end{array}$ & HIP & ST & $B-V$ & $\begin{array}{l}\text { Mass } \\
\left(M_{\odot}\right)\end{array}$ & $\begin{array}{l}\text { Age } \\
(\mathrm{Myr})\end{array}$ & $\begin{array}{c}v \sin i \\
\left(\mathrm{~km} \mathrm{~s}^{-1}\right)\end{array}$ & $\begin{array}{c}\text { Rotation } \\
\text { period (days) }\end{array}$ & $\mathrm{IR} / \mathrm{D}$ \\
\hline HD 105 & 490 & G0V & 0.600 & $1.1^{(a)}$ & $45_{10}^{5(a)}$ & 14.5 & - & $\mathrm{y}^{(b)} / \mathrm{y}^{(b)}$ \\
\hline HD 984 & 1134 & F7V & 0.500 & $1.2^{(a)}$ & $42_{7}^{8(a)}$ & 40.0 & - & $-/ \mathrm{n}^{(c)}$ \\
\hline HD 987 & 1113 & G8V & 0.756 & $0.98^{(d)}$ & $30_{15}^{15}(c)$ & 7.3 & $3.72 \pm 0.01^{(e)}$ & $\mathrm{n}^{(f)} / \mathrm{y}^{(d)}$ \\
\hline HD 1466 & 1481 & F8V & 0.540 & $1.2^{(a)}$ & $45_{10}^{5}(a)$ & 21.0 & - & $\mathrm{y}^{(g)} / \mathrm{y}^{(h)}$ \\
\hline HD 3221 & 2729 & $\mathrm{~K} 4 \mathrm{~V}$ & 1.226 & $0.9^{(e)}$ & $45_{10}^{5}(a)$ & 123.0 & $0.370 \pm 0.002^{(e)}$ & $\mathrm{n}^{(i)} / \mathrm{y}^{(h)}$ \\
\hline HD 6569 & 5191 & K1V & 0.830 & $0.8^{(a)}$ & $149_{49}^{31(a)}$ & 6.0 & $7.13 \pm 0.05^{(e)}$ & $-1-$ \\
\hline HD 7661 & 5938 & K0V & 0.770 & - & $300_{50}^{50}(c)$ & 7.5 & $7.46^{(j)}$ & $\mathrm{n}^{(k)} / \mathrm{n}^{(h)}$ \\
\hline HD 10008 & 7576 & $\mathrm{~K} 0 \mathrm{~V}$ & 0.803 & $0.8^{(a)}$ & $250_{50}^{50(a)}$ & 3.5 & $7.15 \pm 0.10^{(l)}$ & $\mathrm{y}^{(m)} / \mathrm{y}^{(n)}$ \\
\hline HD 16765 & 12530 & F71V & 0.520 & - & - & 32.0 & - & $-1-$ \\
\hline HD 17925 & 13402 & $\mathrm{~K} 1 \mathrm{~V}$ & 0.860 & $0.9^{(a)}$ & $150_{80}^{150(a)}$ & 8.5 & $6.76^{(j)}$ & $\mathrm{y}^{(o)} /-$ \\
\hline HD 18599 & 13754 & $\mathrm{~K} 2 \mathrm{~V}$ & 0.880 & - & - & 4.3 & - & $-1-$ \\
\hline HD 19668 & 14684 & $\mathrm{~K} 0 \mathrm{~V}$ & 0.780 & $0.9^{(a)}$ & $149_{49}^{31(a)}$ & 6.5 & $5.46 \pm 0.08^{(e)}$ & $\mathrm{y}^{(p)} / \mathrm{y}^{(c)}$ \\
\hline HD 24916 & 18512 & $\mathrm{~K} 4 \mathrm{~V}$ & 1.152 & $0.7^{(q)}$ & - & 4.5 & - & $-1-$ \\
\hline HD 25457 & 18859 & F6V & 0.500 & $1.2^{(a)}$ & $149_{49}^{31(a)}$ & 22.0 & $3.13^{(j)}$ & $\mathrm{y}^{(r)} /-$ \\
\hline HD 26923 & 19859 & G0IV & 0.560 & $1.07^{(q)}$ & - & 3.5 & - & $-1-$ \\
\hline HD 29391 & 21547 & FOIV & 0.277 & $1.75^{(s)}$ & $37_{9}^{9}(\mathrm{am})$ & 80.0 & - & $\mathrm{y}^{(n)} /-$ \\
\hline HD 30447 & 22226 & F3V & 0.390 & $1.4^{(a)}$ & $42_{7}^{8(a)}$ & 50.0 & - & $\mathrm{y}^{(t)} / \mathrm{y}^{(u)}$ \\
\hline HD 35650 & 25283 & K6V & 1.311 & $0.7^{(e)}$ & - & 4.0 & $9.34 \pm 0.08^{(e)}$ & $\mathrm{y}^{(r)} / \mathrm{y}^{(v)}$ \\
\hline HD 37572 & 26373 & K0V & 1.094 & $0.9^{(d)}$ & $149_{49}^{31(a)}$ & 9.2 & $4.52 \pm 0.02^{(e)}$ & $\mathrm{y}^{(r)} / \mathrm{n}^{(d)}$ \\
\hline HD 39060 & 27321 & A6V & 0.170 & $1.64^{(d)}$ & $30^{(d)}$ & 130.0 & - & $\mathrm{y}^{(w)} / \mathrm{y}^{(x)}$ \\
\hline HD 41593 & 28954 & $\mathrm{~K} 0 \mathrm{~V}$ & 0.825 & $1.01^{(q)}$ & $329_{93}^{93}(\mathrm{~m})$ & 4.5 & - & $-1-$ \\
\hline HD 43989 & 30030 & G0V & 0.570 & $1.1^{(a)}$ & $45_{10}^{5}(a)$ & 45.5 & $1.15^{(j)}$ & $\mathrm{y}^{(p)} / \mathrm{y}^{(c)}$ \\
\hline HD 44627 & 30034 & K1V & 0.805 & $0.9^{(e)}$ & $30_{15}^{15(c)}$ & 11.5 & $3.85 \pm 0.01^{(e)}$ & $-/ \mathrm{n}^{(c)}$ \\
\hline HD 45081 & 29964 & $\mathrm{~K} 4 \mathrm{~V}$ & 1.251 & $0.8^{(e)}$ & - & 16.4 & $2.67 \pm 0.01^{(e)}$ & $\mathrm{n}^{(y)} / \mathrm{y}^{(c)}$ \\
\hline HD 45270 & 30314 & G1V & 0.602 & $1.11^{(d)}$ & $149_{49}^{31(a)}$ & 17.6 & - & $\mathrm{y}^{(p)} / \mathrm{n}^{(d)}$ \\
\hline HD 59967 & 36515 & G3V & 0.639 & $1.09^{(m)}$ & $353_{58}^{58(m)}$ & 4.0 & - & $\mathrm{y}^{(m)} /-$ \\
\hline HD 61005 & 36948 & G8V & 0.740 & $1.0^{(a)}$ & $50_{10}^{20(a)}$ & 8.5 & $5.04 \pm 0.03^{(a a)}$ & $\mathrm{y}^{(a b)} / \mathrm{y}^{(a c)}$ \\
\hline HD 63608 & 37923 & $\mathrm{~K} 0 \mathrm{~V}$ & 0.830 & $1.0^{(a)}$ & $250_{50}^{50(a)}$ & 3.2 & - & $-1-$ \\
\hline HD 77825 & 44526 & $\mathrm{~K} 2 \mathrm{~V}$ & 0.992 & $0.8^{(a)}$ & $350_{150}^{150(a)}$ & 7.0 & $8.64^{(a d)}$ & $-1-$ \\
\hline HD 82558 & 46816 & $\mathrm{~K} 1 \mathrm{~V}$ & 0.870 & $0.8^{(a e)}$ & - & 29.0 & $1.70^{(j)}$ & $-1-$ \\
\hline HD 89449 & 50564 & F6IV & 0.440 & - & - & 16.0 & - & $-1-$ \\
\hline
\end{tabular}

Notes. Spectral type (ST) and $B-V$ values are taken from the CDS database. The $v \sin i$ values are taken from the CDS if present, or are computed with SAFIR based on the CCF width. The IR/D column report if either an IR excess is reported $(y)$ or not $(n)$ and if a disc has been imaged $(y)$ or not $(n)$ in the literature.

References. ${ }^{(a)}$ Vigan et al. (2017), ${ }^{(b)}$ Meyer et al. (2004), ${ }^{\left({ }^{(c)}\right.}$ Weise et al. (2010), ${ }^{(d)}$ Lagrange et al. (2013), ${ }^{(e)}$ Messina et al. (2010), ${ }^{(f)}$ Rebull et al. (2008), ${ }^{(g)}$ Sierchio et al. (2014), ${ }^{\left({ }^{h}\right.}$ Mamajek et al. (2004), ${ }^{(i)}$ Donaldson et al. (2012), ${ }^{(j)}$ Wright et al. (2011), ${ }^{(k)}$ Lawler et al. (2009), ${ }^{(l)}$ Folsom et al. (2018), ${ }^{(m)}$ Plavchan et al. (2009), ${ }^{(n)}$ Patel et al. (2014), ${ }^{(o)}$ Hillenbrand et al. (2008), ${ }^{(p)}$ Carpenter et al. (2009), ${ }^{(q)}$ Ammler-von Eiff \& Guenther (2009), ${ }^{(r)}$ Zuckerman et al. (2011), ${ }^{\left({ }^{)}\right.}$Simon \& Schaefer (2011), ${ }^{(t)}$ Chen et al. (2014), ${ }^{(u)}$ Soummer et al. $(2014),{ }^{(v)}$ Choquet et al. (2016), ${ }^{(w)}$ Aumann (1985), ${ }^{(x)}$ Smith \& Terrile (1984), ${ }^{(y)}$ McDonald et al. (2012), ${ }^{(a)}$ Folsom et al. (2018), ${ }^{(a b)}$ Meyer et al. (2008), ${ }^{(a c)}$ Meyer et al. (2006), ${ }^{(a d)}$ Kiraga (2012), ${ }^{(a e)}$ Kovári et al. (2004), ${ }^{(a f)}$ Morales et al. (2016), ${ }^{(a g)}$ Chen et al. (2005), ${ }^{\left({ }^{(a h)}\right.}$ Golimowski et al. (2011), ${ }^{(a i)}$ Rameau et al. (2013a), ${ }^{(a j)}$ Meshkat et al. (2013), ${ }^{(a k)}$ Rhee et al. (2007), ${ }^{(a l)}$ Moór et al. (2013), ${ }^{(a m)}$ Montet et al. (2014), ${ }^{(a n)}$ Kiraga (2012), ${ }^{(a o)}$ Mannings \& Barlow (1998), ${ }^{(a p)}$ de la Reza \& Pinzón (2004), ${ }^{(a q)}$ Bailey et al. (2014), ${ }^{(a r)}$ Pecaut et al. (2012), ${ }^{(a s)}$ Sierchio et al. (2014), ${ }^{(a t)}$ Kalas et al. (2004), ${ }^{(a u)}$ Montesinos et al. (2009), ${ }^{(a v)}$ Chauvin et al. (2017b), ${ }^{(a w)}$ Nielsen et al. (2017), ${ }^{(a x)}$ Koen \& Eyer (2002), ${ }^{(a y)}$ Brandt et al. (2014), ${ }^{\left({ }^{(a)}\right)}$ Morales et al. (2016), ${ }^{(b a)}$ Strassmeier et al. (2003), ${ }^{(b b)}$ Messina et al. (2017), ${ }^{(b c)}$ Fuhrmann et al. (2017), ${ }^{(b d)}$ Olmedo et al. (2013), ${ }^{(b e)}$ Schneider et al. (2006), ${ }^{(b f)}$ Bonavita et al. (2016), ${ }^{(b g)}$ Zuckerman et al. (2013), ${ }^{(b h)}$ Desidera et al. (2015), ${ }^{(b i)}$ Churcher et al. (2011), ${ }^{(b j)}$ Rameau et al. (2013b), ${ }^{(b k)}$ Kalas et al. (2004), ${ }^{(b l)}$ Moro-Martín et al. (2015), ${ }^{(b m)}$ Milli et al. (2017), ${ }^{(b n)}$ Delorme et al. (2017), ${ }^{(b o)}$ Beichman et al. (2006), ${ }^{(b p)}$ Mamajek (2012), ${ }^{(b q)}$ Backman \& Gillett (1987), ${ }^{(b r)}$ Holland et al. (1998), ${ }^{(b s)}$ Delorme et al. (2012), ${ }^{(b t)}$ Zuckerman \& Song (2004), ${ }^{(b u)}$ Lindgren \& Heiter (2017), ${ }^{(b v)}$ Passegger et al. (2018), ${ }^{(b w)}$ Poveda et al. (2009), ${ }^{(b x)}$ Morin et al. (2008). 
Table A.1. continued.

\begin{tabular}{|c|c|c|c|c|c|c|c|c|}
\hline $\begin{array}{l}\text { Name } \\
\mathrm{HD} / \mathrm{BD} / \mathrm{CD}\end{array}$ & HIP & ST & $B-V$ & $\begin{array}{l}\text { Mass } \\
\left(M_{\odot}\right)\end{array}$ & $\begin{array}{l}\text { Age } \\
(\mathrm{Myr})\end{array}$ & $\begin{array}{c}v \sin i \\
\left(\mathrm{~km} \mathrm{~s}^{-1}\right)\end{array}$ & $\begin{array}{c}\text { Rotation } \\
\text { period (days) }\end{array}$ & IR/D \\
\hline HD 90905 & 51386 & F5V & 0.569 & $1.16^{(d)}$ & $170_{70}^{180(a)}$ & 10.0 & $2.60^{(j)}$ & $\mathrm{y}^{(p)} / \mathrm{y}^{(a f)}$ \\
\hline HD 92945 & 52462 & K1V & 0.877 & $0.9^{(a)}$ & $170_{70}^{130(a)}$ & 4.0 & - & $\mathrm{n}^{(a g)} / \mathrm{y}^{(a h)}$ \\
\hline HD 95086 & 53524 & A8III & 0.240 & $1.6^{(a i)}$ & $17_{4}^{4(a j)}$ & 22.5 & - & $\mathrm{y}^{(a k)} / \mathrm{y}^{(a l)}$ \\
\hline HD 95650 & 53985 & $\mathrm{M} 2 \mathrm{~V}$ & 1.477 & $0.59^{(\mathrm{am})}$ & - & 11.0 & $14.80^{(a n)}$ & $-/-$ \\
\hline HD 99211 & 55705 & A7V & 0.210 & - & - & 140.0 & - & $\mathrm{y}^{(a o)} /-$ \\
\hline HD 102458 & 57524 & G4V & 0.630 & $1.70^{(a p)}$ & - & 31.0 & - & $\mathrm{y}^{(f)} / \mathrm{n}^{(d)}$ \\
\hline HD 103743 & 58241 & G4V & 0.670 & - & - & 9.0 & - & $-1-$ \\
\hline HD 105690 & 59315 & G5V & 0.661 & $1.02^{(d)}$ & $8_{8}^{15(c)}$ & 8.5 & - & $-1-$ \\
\hline HD 106906 & 59960 & F5V & 0.400 & $1.5^{(a q)}$ & $13_{2}^{2(a r)}$ & 55.0 & - & $\mathrm{y}^{(a s)} / \mathrm{y}^{(a t)}$ \\
\hline HD 108767 & 60965 & K0V & -0.050 & $2.74^{(a u)}$ & - & 6.0 & - & $-1-$ \\
\hline HD 116434 & 65426 & $\mathrm{~A} 2 \mathrm{~V}$ & 0.200 & $1.96^{(a v)}$ & $14_{4}^{4(a v)}$ & 300.0 & - & $\mathrm{n}^{(a v)} /-$ \\
\hline HD 118100 & 66252 & $\mathrm{~K} 5 \mathrm{~V}$ & 1.180 & $0.7^{(a)}$ & $150_{50}^{50(a)}$ & 0.5 & $3.96^{(j)}$ & -I- \\
\hline HD 131399 & 72940 & A1V & 0.080 & $2.08^{(a w)}$ & $21_{3}^{4}(a w)$ & 25.6 & - & $-/-$ \\
\hline HD 141943 & - & G2V & 0.505 & $1.09^{(d)}$ & $30_{15}^{15(c)}$ & 40.0 & $2.2^{(a n)}$ & $-/ \mathrm{n}^{(d)}$ \\
\hline HD 146464 & 79958 & $\mathrm{~K} 3 \mathrm{~V}$ & 1.033 & - & - & 17.0 & $2.329^{(a x)}$ & $-1-$ \\
\hline HD 146624 & 79881 & A0V & 0.022 & $1.9^{(d)}$ & $12^{(d)}$ & 39.0 & - & $-/ \mathrm{n}^{(d)}$ \\
\hline HD 152555 & 82688 & F8V & 0.600 & - & $130_{20}^{20}(a y)$ & 10.0 & $2.77^{(j)}$ & $\mathrm{n}^{(r)} / \mathrm{n}^{(c)}$ \\
\hline HD 159492 & 86305 & A5IV & 0.189 & - & - & 54.0 & - & $-/ y^{(a z)}$ \\
\hline HD 164249 & 88399 & F6V & 0.460 & $1.3^{(a)}$ & $24_{5}^{5(a)}$ & 15.0 & - & $\mathrm{y}^{(t)} /-$ \\
\hline HD 169178 & - & K0V & 0.850 & - & - & 5.5 & - & $-/-$ \\
\hline HD 171488 & 91043 & $\mathrm{G} 2 \mathrm{~V}$ & 0.551 & $1.1^{(a)}$ & $45_{25}^{35}(a)$ & 38.5 & $1.3371 \pm 0.0002^{(b a)}$ & $-/-$ \\
\hline HD 172555 & 92024 & A7V & 0.200 & $1.61^{(d)}$ & $12^{(b b)}$ & 116.5 & - & $\mathrm{y}^{(d)} / \mathrm{y}^{(d)}$ \\
\hline HD 174429 & 92680 & G9IV & 0.878 & $1.2^{(a)}$ & $24_{5}^{5}(a)$ & 69.0 & $0.944 \pm 0.001^{(b b)}$ & $\mathrm{y}^{(t)} /-$ \\
\hline HD 177171 & 93815 & F6V & 0.526 & $1.25^{(a p)}$ & $30^{(d)}$ & 300.0 & $4.737^{(a x)}$ & $-/ \mathrm{n}^{(d)}$ \\
\hline HD 181321 & 95149 & G2V & 0.630 & $0.89^{(b c)}$ & - & 13.0 & $5.7^{(b d)}$ & $-/-$ \\
\hline HD 181327 & 95270 & F6V & 0.460 & $1.36^{(d)}$ & $24_{5}^{5}(a)$ & 19.5 & - & $\mathrm{y}^{(a o)} / \mathrm{y}^{(b e)}$ \\
\hline HD 183414 & 96334 & G3V & 0.650 & $1.04^{(d)}$ & $150_{80}^{70(a)}$ & 9.8 & $3.924^{(a x)}$ & $-1-$ \\
\hline HD 186704 & 97255 & G0V & 0.583 & $1.4^{(b f)}$ & $100^{(b g)}$ & 11.0 & $3.511^{(a n)}$ & $\mathrm{y}^{(y)} /-$ \\
\hline HD 188228 & 98495 & $\mathrm{~A} 0 \mathrm{~V}$ & -0.013 & $2.03^{(e)}$ & $40^{(d)}$ & 85.0 & - & $-/ \mathrm{n}^{(d)}$ \\
\hline HD 189245 & 98470 & F7V & 0.490 & $1.2^{(a)}$ & $150_{50}^{150(a)}$ & 80.0 & $1.88 \pm 0.01^{(b h)}$ & $-1-$ \\
\hline HD 191089 & 99273 & F5V & 0.440 & $1.3^{(a)}$ & $24_{5}^{5}(c)$ & 37.3 & $0.488 \pm 0.005^{(b h)}$ & $\mathrm{y}^{(a o)} / \mathrm{y}^{(b i)}$ \\
\hline HD 197481 & 102409 & M1V & 1.423 & $0.6^{(e)}$ & $21_{5}^{7(a y)}$ & 9.6 & $4.84 \pm 0.02^{(e)}$ & $\mathrm{y}^{(b j)} / \mathrm{y}^{(b k)}$ \\
\hline HD 197890 & 102626 & $\mathrm{~K} 3 \mathrm{~V}$ & 1.053 & $1.0^{(a)}$ & $45_{35}^{55(a)}$ & 140.0 & $0.3804^{(a n)}$ & $\mathrm{y}^{(y)} /-$ \\
\hline HD 202917 & 105388 & G7V & 0.650 & $0.9^{(a)}$ & $45_{10}^{5}(a)$ & 15.4 & $3.36 \pm 0.01^{(e)}$ & $\mathrm{y}^{(n)} / \mathrm{y}^{(u)}$ \\
\hline HD 206860 & 107350 & G0V & 0.580 & $1.1^{(a)}$ & $300_{100}^{700}(a)$ & 12.0 & $4.86^{(j)}$ & $\mathrm{y}^{(t)} / \mathrm{y}^{(b l)}$ \\
\hline HD 206893 & 107412 & F5V & 0.440 & $1.24^{(b m)}$ & $250_{200}^{450(b n)}$ & 29.0 & - & $\mathrm{y}^{(a s)} / \mathrm{y}^{(b m)}$ \\
\hline HD 207575 & 107947 & F6V & 0.490 & $1.24^{(d)}$ & $45_{15}^{5}(a)$ & 30.0 & - & $\mathrm{y}^{(r)} / \mathrm{n}^{(d)}$ \\
\hline HD 213845 & 111449 & F7V & 0.440 & $1.4^{(a)}$ & $250_{50}^{750(a)}$ & 34.0 & - & $\mathrm{y}^{(b o)} /-$ \\
\hline HD 215641 & 112491 & G8V & 0.760 & - & $440_{40}^{40(a y)}$ & 3.6 & - & $-1-$ \\
\hline HD 216956 & 113368 & A3V & 0.090 & $1.92^{(b p)}$ & $440_{40}^{40}(b p)$ & 90.0 & - & $\mathrm{y}^{(b q)} / \mathrm{y}^{(b r)}$ \\
\hline HD 217343 & 113579 & G5V & 0.640 & $1.05^{(a)}$ & $70^{(d)}$ & 12.4 & - & $-/ \mathrm{n}^{(d)}$ \\
\hline HD 217987 & 114046 & $\mathrm{M} 2 \mathrm{~V}$ & 1.480 & $0.47^{(\mathrm{am})}$ & $100--10000^{(b s)}$ & 2.5 & - & $-/-$ \\
\hline HD 218396 & 114189 & $\mathrm{~A} 5 \mathrm{~V}$ & 0.257 & $1.46^{(a)}$ & $30^{(d)}$ & 49.0 & - & $\mathrm{y}^{(b t)} / \mathrm{y}^{(a k)}$ \\
\hline HD 218860 & 114530 & G8V & 0.738 & $1.0^{(e)}$ & - & 6.6 & $5.17 \pm 0.02^{(e)}$ & $\mathrm{y}^{(r)} /-$ \\
\hline
\end{tabular}


Table A.1. continued.

\begin{tabular}{|c|c|c|c|c|c|c|c|c|}
\hline $\begin{array}{l}\text { Name } \\
\mathrm{HD} / \mathrm{BD} / \mathrm{CD}\end{array}$ & HIP & ST & $B-V$ & $\begin{array}{l}\text { Mass } \\
\left(M_{\odot}\right)\end{array}$ & $\begin{array}{l}\text { Age } \\
(\mathrm{Myr})\end{array}$ & $\begin{array}{c}v \sin i \\
\left(\mathrm{~km} \mathrm{~s}^{-1}\right)\end{array}$ & $\begin{array}{c}\text { Rotation } \\
\text { period (days) }\end{array}$ & $\mathrm{IR} / \mathrm{D}$ \\
\hline HD 221575 & 116258 & $\mathrm{~K} 2 \mathrm{~V}$ & 0.930 & - & - & 3.6 & - & $-1-$ \\
\hline HD 223340 & - & K1V & 0.820 & - & - & 7.0 & - & $-1-$ \\
\hline HD 224228 & 118008 & $\mathrm{~K} 2 \mathrm{~V}$ & 0.985 & $0.86^{(d)}$ & $149_{49}^{31(a)}$ & 2.6 & - & $\mathrm{y}^{(r)} / \mathrm{n}^{(d)}$ \\
\hline- & 6276 & G9V & 0.800 & $0.9^{(a)}$ & $149_{49}^{31(a)}$ & 10.0 & $6.40^{(j)}$ & $\mathrm{y}^{(a s)} / \mathrm{y}^{(c)}$ \\
\hline- & 116384 & $\mathrm{~K} 7 \mathrm{~V}$ & 1.347 & - & - & 4.5 & - & $-1-$ \\
\hline- & 17157 & K7V & 1.300 & - & - & 5.0 & - & $-1-$ \\
\hline- & 23309 & MOV & 1.471 & $0.55^{(a p)}$ & $10_{3}^{3(c)}$ & 5.8 & $8.60 \pm 0.07^{(e)}$ & $-/ \mathrm{n}^{(f)}$ \\
\hline- & 31878 & $\mathrm{~K} 7 \mathrm{~V}$ & 1.296 & $0.643^{(b u)}$ & - & 5.0 & $9.06 \pm 0.08^{(e)}$ & $-1-$ \\
\hline- & 36985 & $\mathrm{M} 2 \mathrm{~V}$ & 1.476 & $0.621^{(b v)}$ & $260_{260}^{420(b w)}$ & 5.0 & $12.16^{(a n)}$ & $-1-$ \\
\hline- & 44722 & K7V & 1.450 & $0.638^{(b u)}$ & - & 9.0 & - & $-1-$ \\
\hline- & 46634 & G5V & 0.860 & - & - & 6.0 & $3.05 \pm 0.03^{(e)}$ & $-1-$ \\
\hline- & 51317 & $\mathrm{M} 2 \mathrm{~V}$ & 1.501 & - & $130_{20}^{40(a y)}$ & 2.6 & - & $-1-$ \\
\hline $\mathrm{BD}+202465$ & - & M5V & 1.300 & $0.42^{(b x)}$ & - & 3.8 & $2.60^{(j)}$ & $-1-$ \\
\hline CD-46 1064 & - & $\mathrm{K} 3 \mathrm{~V}$ & 1.048 & $0.8^{(a)}$ & $45_{10}^{5}(a)$ & 1.0 & $3.74 \pm 0.04^{(e)}$ & $-1-$ \\
\hline
\end{tabular}

Table A.2. Results for the 89 stars of our HARPS RV survey.

\begin{tabular}{|c|c|c|c|c|c|c|c|c|c|c|c|c|c|c|}
\hline \multicolumn{3}{|c|}{ Stellar characteristics } & \multicolumn{12}{|c|}{ Survey results. } \\
\hline \multirow{3}{*}{$\begin{array}{l}\text { Name } \\
\mathrm{HD} / \mathrm{BD} / \mathrm{CD}\end{array}$} & \multirow{3}{*}{ HIP } & \multirow{3}{*}{$\begin{array}{c}\mathrm{ST} \\
\text { (day) }\end{array}$} & \multirow[t]{3}{*}{ TBL } & \multirow[t]{3}{*}{$N_{\mathrm{m}}$} & \multicolumn{3}{|c|}{$\mathrm{RV}$} & \multicolumn{3}{|c|}{ BVS } & \multirow{3}{*}{$\begin{array}{l}\text { RV- } \\
\text { BVS } \\
\text { corr. }\end{array}$} & \multirow{3}{*}{\multicolumn{2}{|c|}{$\begin{array}{rr}\langle F W H M\rangle & \left\langle\log R_{\mathrm{HK}}^{\prime}\right\rangle \\
& \left(\mathrm{km} \mathrm{s}^{-1}\right)\end{array}$}} & \multirow[t]{3}{*}{$\mathrm{V}$} \\
\hline & & & & & $A$ & $\mathrm{rms}$ & $\langle U\rangle$ & $A$ & $\mathrm{rms}$ & $\langle U\rangle$ & & & & \\
\hline & & & & & & $\left(\mathrm{ms}^{-1}\right)$ & & & $\left(\mathrm{ms}^{-1}\right.$ & & & & & \\
\hline HD 105 & 490 & G0V & 4606 & 36 & 236.8 & 61.1 & 4.2 & 310.7 & 9.8 & 72.9 & -0.67 & 22.6 & -4.316 & \\
\hline HD 984 & 1134 & F7V & 867 & 21 & 301.9 & 84.6 & 16.4 & 571.8 & 38.2 & 137.4 & -0.46 & 59.7 & -4.368 & \\
\hline HD 987 & 1113 & G8V & 2621 & 19 & 502.6 & 116.8 & 2.5 & 393.6 & 6.4 & 103.1 & -1.08 & 13.0 & -4.088 & \\
\hline HD 1466 & 1481 & F8V & 4400 & 19 & 135.8 & 39.6 & 7.4 & 189.8 & 17.6 & 55.9 & -0.66 & 32.3 & -4.344 & \\
\hline HD 3221 & 2729 & $\mathrm{~K} 4 \mathrm{~V}$ & 4014 & 5 & 4793.4 & 1794.3 & 68.6 & 3115.1 & 172.5 & 1447.8 & -0.54 & 203.6 & -4.474 & \\
\hline HD 6569 & 5191 & K1V & 8 & 4 & 24.0 & 11.4 & 1.5 & 17.8 & 3.8 & 7.5 & -1.49 & 9.6 & -4.228 & \\
\hline HD 7661 & 5938 & $\mathrm{~K} 0 \mathrm{~V}$ & 1525 & 29 & 96.0 & 28.1 & 1.2 & 50.5 & 3.3 & 12.5 & -1.42 & 9.2 & -4.325 & \\
\hline HD 10008 & 7576 & $\mathrm{~K} 0 \mathrm{~V}$ & 4021 & 17 & 20.5 & 6.2 & 1.0 & 17.5 & 2.7 & 5.2 & 0.44 & 7.5 & -4.358 & \\
\hline HD 16765 & 12530 & F71V & 926 & 27 & 173.0 & 47.2 & 11.0 & 402.9 & 27.9 & 93.1 & -0.42 & 48.5 & -4.475 & \\
\hline HD 17925 & 13402 & K1V & 1470 & 40 & 113.6 & 30.5 & 1.1 & 58.7 & 3.1 & 17.4 & -0.87 & 10.0 & -4.310 & \\
\hline HD 18599 & 13754 & $\mathrm{~K} 2 \mathrm{~V}$ & 1055 & 16 & 115.9 & 38.9 & 1.5 & 54.3 & 3.9 & 17.6 & -1.46 & 9.8 & -4.310 & \\
\hline HD 19668 & 14684 & KOV & 4402 & 20 & 143.1 & 33.2 & 1.8 & 91.9 & 4.7 & 26.0 & -0.95 & 11.8 & -4.284 & \\
\hline HD 24916 & 18512 & $\mathrm{~K} 4 \mathrm{~V}$ & 792 & 22 & 24.3 & 7.4 & 0.8 & 36.7 & 2.2 & 11.6 & 0.46 & 7.8 & -4.536 & \\
\hline HD 25457 & 18859 & F6V & 4089 & 78 & 187.2 & 49.5 & 3.3 & 345.4 & 7.7 & 60.2 & -0.66 & 28.2 & -4.355 & \\
\hline HD 26923 & 19859 & G0IV & 4010 & 47 & 50.2 & 12.7 & 1.1 & 24.7 & 3.1 & 5.6 & 1.12 & 8.0 & -4.487 & \\
\hline HD 29391 & 21547 & FOIV & 3996 & 81 & 1476.1 & 326.2 & 28.4 & 2564.3 & 72.1 & 487.8 & - & 101.4 & & \\
\hline HD 30447 & 22226 & F3V & 792 & 19 & 194.1 & 47.2 & 47.7 & 7048.7 & 106.6 & 1728.3 & - & 120.7 & -4.447 & \\
\hline HD 35650 & 25283 & K6V & 1195 & 13 & 48.3 & 15.3 & 1.2 & 43.6 & 3.0 & 12.3 & -0.67 & 9.4 & -4.359 & \\
\hline HD 37572 & 26373 & $\mathrm{KOV}$ & 2826 & 34 & 347.1 & 75.4 & 1.7 & 223.6 & 4.4 & 52.8 & -1.28 & 15.6 & -4.165 & \\
\hline HD 39060 & 27321 & A6V & 3702 & 5108 & 755.5 & 341.4 & 58.9 & & & & - & & & \\
\hline HD 41593 & 28954 & $\mathrm{~K} 0 \mathrm{~V}$ & 1194 & 15 & 69.5 & 19.8 & 1.1 & 59.4 & 2.9 & 16.7 & -0.66 & 9.1 & -4.370 & \\
\hline HD 43989 & 30030 & G0V & 4433 & 17 & 819.0 & 238.6 & 15.6 & 743.7 & 34.3 & 197.6 & -0.83 & 64.2 & -4.209 & \\
\hline
\end{tabular}

Notes. Spectral type (ST) are taken from the CDS database. The survey results include the time baseline (TBL), the number of computed spectra $N_{\mathrm{m}}$, the amplitude corresponding to the difference between the maximum and the minimum of the $\operatorname{RV}(A)$, rms and mean uncertainty $\langle U\rangle$ on the $\mathrm{RV}$ and BVS measurements, the RV-BVS correlation factor (slope of the best linear fit), the mean FWHM ( $\langle\mathrm{FWHM}\rangle)$, and the mean $\log R_{\mathrm{HK}}^{\prime}$ $\left(\left\langle\log R_{\mathrm{HK}}^{\prime}\right\rangle\right)$. V stands for the dominant source of RV variations, with A for stellar activity (spots), $\mathrm{P}$ for pulsations, $\mathrm{B}$ for binary and, $\mathrm{T}$ for long-term trend. 
Table A.2. continued.

\begin{tabular}{|c|c|c|c|c|c|c|c|c|c|c|c|c|c|c|}
\hline \multicolumn{3}{|c|}{ Stellar characteristics } & \multicolumn{12}{|c|}{ Survey results. } \\
\hline \multirow{3}{*}{$\begin{array}{l}\text { Name } \\
\mathrm{HD} / \mathrm{BD} / \mathrm{CD}\end{array}$} & \multirow{3}{*}{ HIP } & \multirow{3}{*}{$\begin{array}{c}\text { ST } \\
\text { (day) } \\
\end{array}$} & \multirow[t]{3}{*}{ TBL } & \multirow[t]{3}{*}{$N_{\mathrm{m}}$} & \multicolumn{3}{|c|}{$\overbrace{}^{R V}$} & \multicolumn{3}{|c|}{ BVS } & \multirow{3}{*}{$\begin{array}{l}\text { RV- } \\
\text { BVS } \\
\text { corr. }\end{array}$} & \multirow{3}{*}{$\langle F W H M\rangle$} & \multirow{3}{*}{$\begin{array}{r}\left\langle\log R_{\mathrm{HK}}^{\prime}\right\rangle \\
\left(\mathrm{km} \mathrm{s}^{-1}\right)\end{array}$} & \multirow[t]{3}{*}{ V } \\
\hline & & & & & $A$ & $\mathrm{rms}$ & $\langle U\rangle$ & $A$ & rms & $\langle U\rangle$ & & & & \\
\hline & & & & & & $\left(\mathrm{ms}^{-1}\right)$ & & & $\left(\mathrm{ms}^{-1}\right.$ & & & & & \\
\hline HD 44627 & 30034 & $\mathrm{~K} 1 \mathrm{~V}$ & 4892 & 23 & 670.6 & 177.7 & 3.2 & 428.6 & 8.1 & 110.9 & - & 19.1 & -4.018 & \\
\hline HD 45081 & 29964 & K4V & 020 & 17 & 852.4 & 252.1 & 5.0 & 911.0 & 13.0 & 246.9 & - & 24.8 & -3.887 & \\
\hline HD 45270 & 30314 & G1V & 2827 & 19 & 137.4 & 51.8 & 3.1 & 193.3 & 8.4 & 59.2 & -0.76 & 27.6 & -4.335 & \\
\hline HD 59967 & 36515 & G3V & 1065 & 25 & 65.3 & 19.7 & 1.4 & 41.6 & 3.6 & 10.5 & -0.99 & 9.0 & -4.328 & \\
\hline HD 61005 & 36948 & G8V & 2369 & 33 & 186.5 & 48.8 & 2.5 & 127.4 & 6.3 & 32.7 & -1.16 & 14.5 & -4.261 & \\
\hline HD 63608 & 37923 & $\mathrm{KOV}$ & 1069 & 36 & 80.7 & 22.2 & 1.1 & 49.1 & 3.0 & 11.7 & -0.63 & 8.0 & -4.323 & \\
\hline HD 77825 & 44526 & $\mathrm{~K} 2 \mathrm{~V}$ & 283 & 6 & 47.0 & 18.3 & 1.4 & 46.5 & 3.4 & 14.9 & -1.09 & 9.6 & -4 & \\
\hline HD 82558 & 46816 & K1V & 1092 & 24 & 296.0 & 79.7 & 7.1 & 475.6 & 17.5 & 124.1 & -0.43 & 42.0 & -3.990 & \\
\hline HD 89449 & 50564 & F6IV & 1227 & 28 & 129.9 & 41.4 & 4.3 & 351.6 & 9.1 & 113.6 & -0.26 & 25.3 & -4.971 & \\
\hline HD 90905 & 51386 & F5V & 2551 & 24 & 125.6 & 40.8 & 1.9 & 187.7 & 5.0 & 49.0 & -0.74 & 15.0 & -4.345 & \\
\hline HD 92945 & 52462 & K1V & 4533 & 38 & 182.3 & 40.5 & 1.5 & 86.2 & 3.9 & 23.8 & -1.37 & 10.3 & -4.261 & \\
\hline HD 95086 & 53524 & A8III & 1532 & 103 & 1279.0 & 268.8 & 14.8 & 2594.0 & 36.3 & 511.1 & - & 45.4 & & \\
\hline HD 95650 & 53985 & $\mathrm{M} 2 \mathrm{~V}$ & 4046 & 12 & 23.8 & 7.6 & 3.1 & 46.1 & 7.3 & 13.6 & - & 8.0 & -4.330 & \\
\hline HD 99211 & 55705 & A7V & 3068 & 112 & 818.0 & 149.4 & 86.8 & 49704.0 & 214.7 & 5222.3 & - & 183.4 & & \\
\hline HD 102458 & 57524 & G4V & 2964 & 26 & 38.5 & 307.7 & 10.0 & 1358.4 & 23.8 & 431.7 & - & 43.6 & -3.994 & \\
\hline HD 103743 & 58241 & G4V & 1065 & 30 & 102.2 & 32.1 & 2.6 & 141.6 & 6.6 & 35.1 & -0.79 & 14.9 & -4.308 & \\
\hline HD 105690 & 59315 & G5V & 2975 & 133 & 269.4 & 61.3 & 2.3 & 193.7 & 5.4 & 44.0 & -0.91 & 15.1 & -4.276 & \\
\hline HD 106906 & 59960 & F5V & 1230 & 46 & 5290.4 & 1285.7 & 32.9 & 9949.8 & 83.3 & 2272.4 & - & 74.0 & -4.574 & \\
\hline HD 108767 & 60965 & $\mathrm{~K} 0 \mathrm{~V}$ & 1201 & 18 & 47.9 & 12.4 & 0.7 & 25.3 & 2.0 & 7.7 & 0.21 & 8.2 & -4.381 & \\
\hline HD 116434 & 65426 & $\mathrm{~A} 2 \mathrm{~V}$ & 439 & 58 & 6273.2 & 1223.2 & 1235.6 & & & & - & & & \\
\hline HD 118100 & 66252 & $\mathrm{~K} 5 \mathrm{~V}$ & 720 & 10 & 534.7 & 155.6 & 6.4 & 139.5 & 15.6 & 43.0 & -3.48 & 16.5 & -4.108 & \\
\hline HD 131399 & 72940 & A1V & 189 & 87 & 19478.2 & 6381.7 & & 1287.6 & 39.3 & 237.0 & - & 43.1 & & \\
\hline HD 141943 & - & G2V & 2648 & 58 & 861.4 & 222.8 & & 1169.7 & 25.3 & 269.6 & -0.66 & 53.0 & -4.02 & \\
\hline HD 146464 & 79958 & K3V & 51 & 5 & 2.1 & 352.1 & & 546.2 & 12.3 & 220.6 & -1.55 & 25.8 & -4.163 & \\
\hline HD 146624 & 79881 & A0V & 4762 & 335 & 232.7 & 39.8 & 29.2 & 70472.6 & 72.9 & 6163.4 & - & 65.1 & & \\
\hline HD 152555 & 82688 & F8V & 1135 & 22 & 225.0 & 64.8 & 6.1 & 283.2 & 15.9 & 69.9 & -0.74 & 26.3 & -4.294 & \\
\hline HD 159492 & 86305 & A5IV & 4751 & 90 & 612.4 & 124.9 & 20.8 & 2413.2 & 52.1 & 359.7 & - & 79.4 & & \\
\hline HD 164249 & 88399 & F6V & 1113 & 25 & & 14.6 & & 134.2 & 17.0 & 36.7 & -0.24 & 29.8 & -4.765 & \\
\hline HD 169178 & - & $\mathrm{KOV}$ & 1123 & 19 & 54.7 & 135.7 & 1.8 & 85.6 & 4.7 & 25.0 & - & 10. & -4.234 & \\
\hline HD 171488 & 91043 & G2V & 1111 & 18 & 2190.4 & 717.7 & 13.1 & 2249.5 & 33.2 & 680.4 & -1.0 & 58.2 & -3.988 & \\
\hline HD 172555 & 92024 & A7V & 2975 & 262 & 1883.8 & 329.6 & 70.6 & 94415.3 & 185.8 & 6148.5 & - & 172.4 & & \\
\hline HD 174429 & 92680 & G9IV & 4572 & 42 & 3362.3 & 970.2 & 26.0 & 1167.7 & 60.0 & 320.7 & -1.77 & 109.9 & & \\
\hline HD 177171 & 93815 & F6V & 144 & 21 & 21233.2 & 5931.1 & 53.4 & & & & - & & -4.047 & \\
\hline HD 181321 & 95149 & $\mathrm{G} 2 \mathrm{~V}$ & 3757 & 28 & 2610.2 & 683.2 & 3.9 & 244.0 & 9.6 & 59.1 & - & 19. & -4.243 & \\
\hline HD 181327 & 95270 & F6V & 3496 & 56 & & 16.3 & & 156.8 & 9.0 & 30.6 & -0.26 & 28 & -4.577 & \\
\hline HD 183414 & 96334 & G3V & 3097 & 68 & 247.9 & 63.6 & 2.4 & 214.0 & 6.2 & & -1.01 & 16. & -4.236 & \\
\hline HD 186704 & 97255 & G0V & 451 & 4 & 345.8 & 168.8 & 4.7 & 77.1 & 11.9 & 29.3 & - & 22.3 & -4.308 & \\
\hline HD 188228 & 98495 & $\mathrm{~A} 0 \mathrm{~V}$ & 4315 & 194 & 1398.7 & 319.2 & 150.2 & 211414.9 & 368.1 & 18294.0 & - & 148.0 & & \\
\hline HD 189245 & 98470 & F7V & 4285 & 46 & 646.2 & 119.4 & 21.0 & 5149.4 & 43.1 & 757.5 & - & 104.2 & -4.304 & \\
\hline HD 191089 & 99273 & F5V & 1025 & 26 & & 22.8 & & 300.0 & 37.3 & 78.4 & - & 57.0 & -4.526 & \\
\hline HD 197481 & 102409 & M1V & 5619 & 55 & & 144.0 & & 470.1 & 8.0 & 101.2 & -1.37 & 16.2 & -4.289 & \\
\hline HD 197890 & 102626 & K3V & 13 & 3 & 922.1 & 376.6 & 85.2 & 3975.6 & 217.1 & 1713.8 & - & 216.2 & -4.277 & \\
\hline HD 202917 & 105388 & G7V & 4438 & 20 & 436.1 & 124.6 & 5.0 & 438.1 & 12.4 & 134.8 & -0.84 & 23.0 & -3.984 & \\
\hline HD 206860 & 107350 & G0V & 1332 & 22 & 122.1 & 35.8 & 2.8 & 180.9 & 6.7 & 51.4 & - & 16.1 & -4.387 & \\
\hline HD 206893 & 107412 & F5V & 542 & 15 & & 45.5 & 10.8 & 361.3 & 21.3 & 68.2 & - & 50.7 & -4.630 & \\
\hline HD 207575 & 107947 & F6V & 2391 & 39 & 227.6 & 49.3 & 9.5 & 389.5 & 19.9 & 97.6 & - & 49.1 & -4.332 & \\
\hline HD 213845 & 111449 & F7V & 4436 & 79 & 220.4 & 44.2 & 9.6 & 454.3 & 25.8 & 82.6 & - & 52.3 & -4.703 & \\
\hline HD 215641 & 112491 & G8V & 1639 & 78 & 101.5 & 20.5 & 1.2 & 84.4 & 3.4 & 12.2 & 0.22 & 8.3 & -4.460 & \\
\hline HD 216956 & 113368 & A3V & 4929 & 834 & 382.4 & 44.3 & 27.0 & & & & - & & & \\
\hline HD 217343 & 113579 & G5V & 2727 & 26 & 276.6 & 96.9 & 2.7 & 298.7 & 6.6 & 85.6 & -1.08 & 19.3 & -4.215 & \\
\hline HD 217987 & 114046 & M2V & 5109 & 130 & 63.8 & 18.5 & 0.9 & 39.5 & 2.3 & 8.2 & 0.95 & 5.8 & -4.899 & \\
\hline HD 218396 & 114189 & A5V & 2727 & 124 & 3616.3 & 924.5 & 38.5 & 7376.0 & 95.5 & 2191.3 & - & 62.3 & & \\
\hline HD 218860 & 114530 & G8V & 1137 & 18 & 137.1 & 36.2 & 2.1 & 82.8 & 5.4 & 18.6 & -1.3 & 11.7 & -4.240 & \\
\hline HD 221575 & 116258 & $\mathrm{~K} 2 \mathrm{~V}$ & 869 & 16 & 45.7 & 12.2 & 1.4 & 33.4 & 3.7 & 12.0 & -0.54 & 8.8 & -4.438 & \\
\hline
\end{tabular}


Table A.2. continued.

\begin{tabular}{|c|c|c|c|c|c|c|c|c|c|c|c|c|c|c|}
\hline \multicolumn{3}{|c|}{ Stellar characteristics } & \multicolumn{12}{|c|}{ Survey results. } \\
\hline \multirow{3}{*}{$\begin{array}{l}\text { Name } \\
\mathrm{HD} / \mathrm{BD} / \mathrm{CD}\end{array}$} & \multirow[t]{3}{*}{ HIP } & \multirow{3}{*}{$\begin{array}{c}\text { ST } \\
\text { (day) }\end{array}$} & \multirow[t]{3}{*}{ TBL } & \multirow[t]{3}{*}{$N_{\mathrm{m}}$} & \multicolumn{3}{|c|}{ RV } & \multicolumn{3}{|c|}{ BVS } & \multirow{3}{*}{$\begin{array}{l}\text { RV- } \\
\text { BVS } \\
\text { corr. }\end{array}$} & \multirow{3}{*}{$\langle\mathrm{FWHM}\rangle$} & \multirow[t]{3}{*}{$\left\langle\log R_{\mathrm{HK}}^{\prime}\right\rangle$} & \multirow[t]{2}{*}{$\overline{\mathrm{V}}$} \\
\hline & & & & & $A$ & $\mathrm{rms}$ & $\langle U\rangle$ & $A$ & $\mathrm{rms}$ & $\langle U\rangle$ & & & & \\
\hline & & & & & \multicolumn{3}{|c|}{$\left(\mathrm{ms}^{-1}\right)$} & \multicolumn{3}{|c|}{$\left(\mathrm{ms}^{-1}\right)$} & & & & \\
\hline HD 223340 & - & $\mathrm{K} 1 \mathrm{~V}$ & 868 & 10 & 54.4 & 17.3 & 1.7 & 42.2 & 4.4 & 10.7 & -1.34 & 9.8 & -4.337 & A \\
\hline HD 224228 & 118008 & $\mathrm{~K} 2 \mathrm{~V}$ & 2815 & 31 & 35.4 & 8.8 & 1.0 & 31.5 & 2.8 & 7.5 & 0.32 & 7.8 & -4.356 & A \\
\hline- & 6276 & G9V & 1201 & 20 & 95.6 & 27.3 & 1.6 & 91.0 & 4.2 & 27.6 & -0.65 & 9.4 & -4.321 & A \\
\hline- & 116384 & $\mathrm{~K} 7 \mathrm{~V}$ & 733 & 8 & 104.3 & 41.2 & 1.7 & 60.2 & 4.5 & 19.2 & -1.18 & 8.6 & -4.455 & A \\
\hline- & 17157 & $\mathrm{~K} 7 \mathrm{~V}$ & 1457 & 6 & 67.5 & 22.3 & 1.4 & 37.1 & 3.6 & 14.9 & - & 8.1 & -4.577 & A \\
\hline- & 23309 & MOV & 4030 & 16 & 245.4 & 64.1 & 2.3 & 116.1 & 5.9 & 33.0 & -1.83 & 11.7 & -3.816 & A \\
\hline- & 31878 & $\mathrm{~K} 7 \mathrm{~V}$ & 435 & 11 & 84.3 & 31.6 & 1.6 & 48.3 & 4.1 & 16.1 & -1.86 & 8.5 & -4.276 & A \\
\hline- & 36985 & $\mathrm{M} 2 \mathrm{~V}$ & 789 & 20 & 670.1 & 247.4 & 2.1 & 58.8 & 5.4 & 14.7 & - & 8.1 & -4.316 & B \\
\hline- & 44722 & K7V & 90 & 4 & 8.0 & 3.4 & 1.5 & 10.7 & 3.8 & 4.0 & -0.49 & 7.6 & -4.550 & A \\
\hline- & 46634 & G5V & 281 & 3 & 38.2 & 16.1 & 1.4 & 32.6 & 3.5 & 13.9 & -1.0 & 9.8 & -4.321 & A \\
\hline- & 51317 & M2V & 4541 & 139 & 17.7 & 3.6 & 1.2 & 24.5 & 3.2 & 3.5 & -0.38 & 4.8 & -5.032 & A \\
\hline $\mathrm{BD}+202465$ & - & M5V & 3983 & 40 & 63.5 & 16.1 & 1.2 & 33.6 & 3.1 & 6.5 & -0.61 & 6.7 & -4.026 & A \\
\hline CD-46 1064 & - & $\mathrm{K} 3 \mathrm{~V}$ & 1185 & 12 & 477.8 & 134.9 & 8.7 & 211.2 & 21.6 & 68.7 & -1.53 & 17.4 & -4.204 & A \\
\hline
\end{tabular}

\section{Appendix B: RV correction from the RV-BVS correlation}

Solar to late-type stars present spots on their surfaces. These spots induce a quasi-periodic variations in the line profiles, which cause a signal in the RV and in the BVS. When the lines are resolved, there is at first order a correlation between the RV and the BVS (see Desort et al. 2007 for a detailed analysis).

To correct the RV signal induced by spots, we use Melo et al. (2007) method. It consists in correcting the RV from the linear regression of the RV vs. BVS dataset. The new RV at a given date $t$ is:

$R V_{\text {corr }}(t)=R V(t)-(a * \operatorname{BVS}(t)+b)$

where $a$ and $b$ are the slope and the intercept of the best linear fit of the RV vs. BVS dataset.

We present in Fig. B.1 an example of such correction for a star with $v \sin i=31 \mathrm{~km} \mathrm{~s}^{-1}$. The mean RV rms of $300 \mathrm{~ms}^{-1}$ are reduced to $60 \mathrm{~ms}^{-1}$ after correction. This method was used in precedent works that use SAFIR (Lagrange et al. 2013; Borgniet et al. 2017, 2019).

We present a star for which a significant offset due to the HARPS fiber change is present in the corrected RV in Fig. B.2. The initial mean RV rms are $36 \mathrm{~ms}^{-1}$. After correction of the offset and of the RV-BVS correlation, the mean RV rms are $10 \mathrm{~ms}^{-1}$.

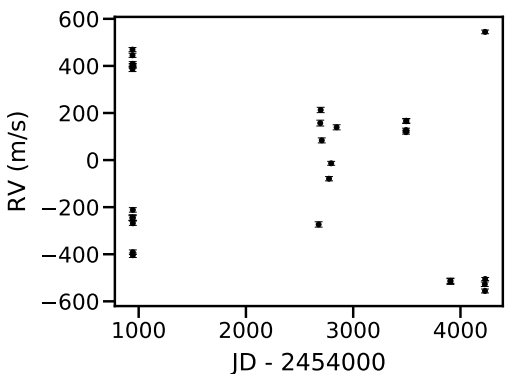

(a)

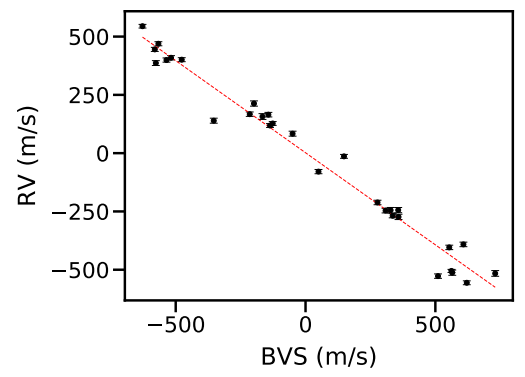

(b)

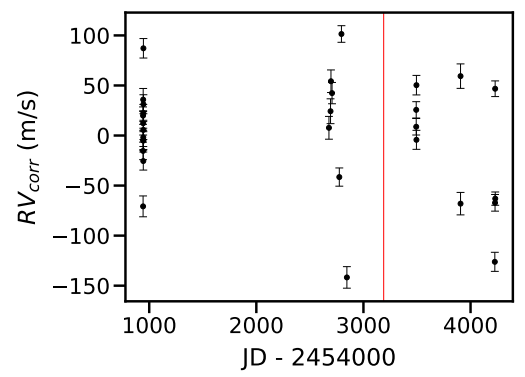

(c)

Fig. B.1. HD $102458 \mathrm{RV}$ jitter correction. (a) RV time variations. (b) RV vs. BVS. The best linear fit is presentend in red dashed line. (c) RV corrected from the RV-BVS correlation. HARPS fiber change is shown with a vertical red line. 


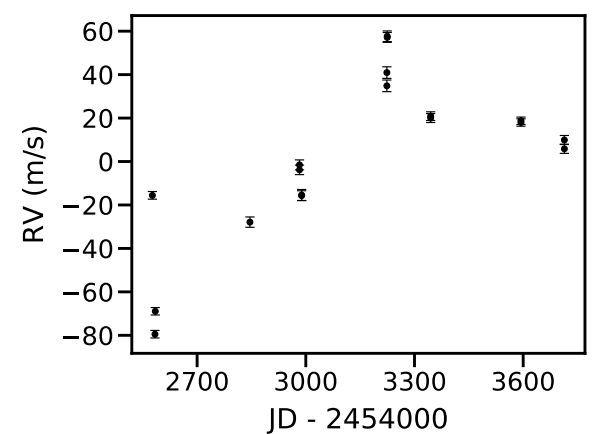

(a)

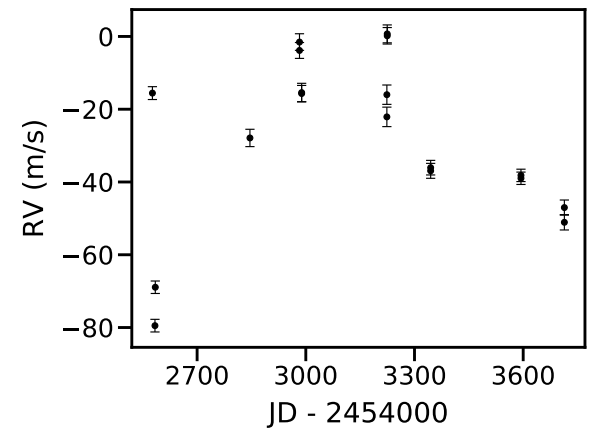

(d)

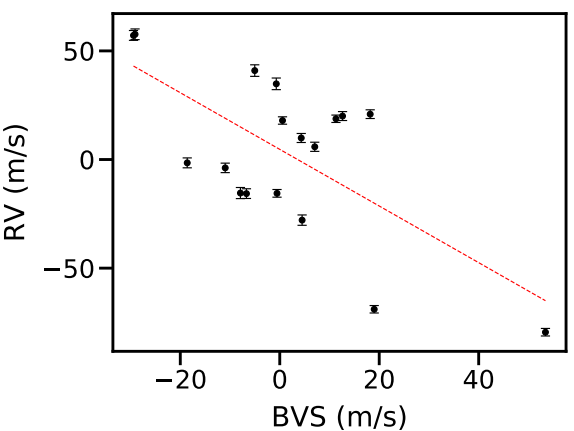

(b)

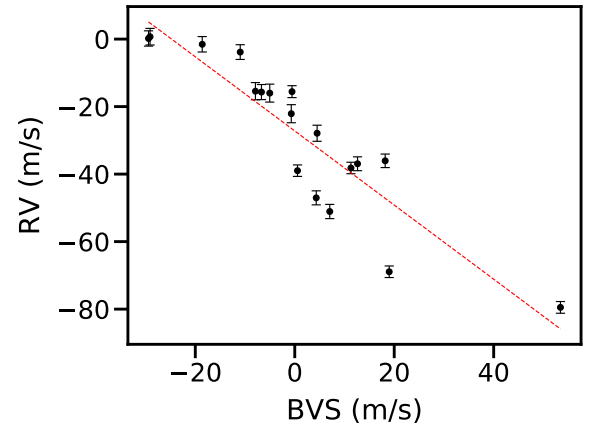

(e)

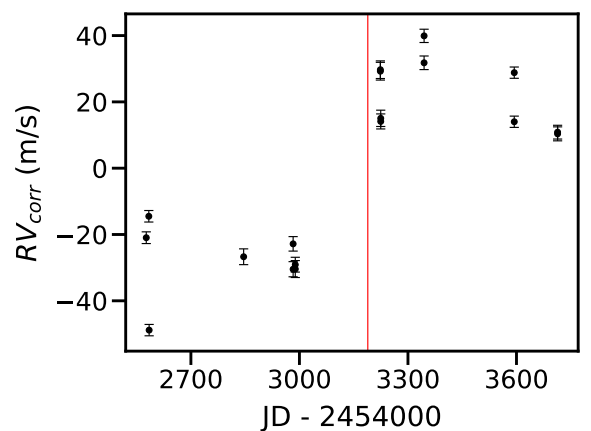

(c)

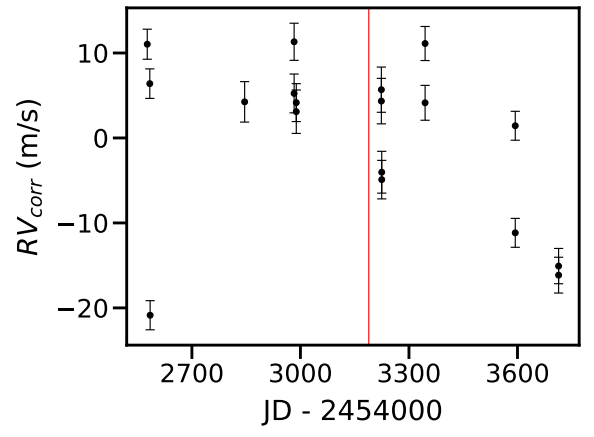

(f)

Fig. B.2. HD 218860 jitter and offset correction. (a) RV time variations. (b) RV vs. BVS. The best linear fit is presented in red dashed line. (c) RV corrected from the RV-BVS correlation. HARPS fiber change is shown with a vertical red line. (d) RV time variations corrected from the offset due to the HARPS fiber change. (e) RV corrected from offset vs. BVS. The best linear fit is presented in red dashed line. $(f)$ RV corrected from offset, corrected from their correlation to the BVS. HARPS fiber change is shown with a vertical red line. 